\title{
Square integrable holomorphic functions on infinite-dimensional Heisenberg type groups
}

\author{
Bruce K. Driver • Maria Gordina
}

Received: 6 August 2008 / Revised: 7 February 2009 / Published online: 1 April 2009 (C) The Author(s) 2009. This article is published with open access at Springerlink.com

\begin{abstract}
We introduce a class of non-commutative, complex, infinite-dimensional Heisenberg like Lie groups based on an abstract Wiener space. The holomorphic functions which are also square integrable with respect to a heat kernel measure $\mu$ on these groups are studied. In particular, we establish a unitary equivalence between the square integrable holomorphic functions and a certain completion of the universal enveloping algebra of the "Lie algebra" of this class of groups. Using quasi-invariance of the heat kernel measure, we also construct a skeleton map which characterizes globally defined functions from the $L^{2}(v)$-closure of holomorphic polynomials by their values on the Cameron-Martin subgroup.
\end{abstract}

Keywords Heisenberg group · Holomorphic · Heat kernel · Quasi-invariance · Taylor map

Mathematics Subject Classification (2000) Primary 35K05 · 43A15; Secondary $58 \mathrm{G} 32$

B. K. Driver's research was supported in part by NSF Grants DMS-0504608 and DMS-0804472 and the Miller Institute at the University of California, at Berkeley.

M. Gordina's research was supported in part by NSF Grant DMS-0706784 and the Humboldt Foundation Research Fellowship.

B. K. Driver $(\bowtie)$

Department of Mathematics, 0112, University of California, San Diego,

La Jolla, CA 92093-0112, USA

e-mail: driver@euclid.ucsd.edu

M. Gordina

Department of Mathematics, University of Connecticut, Storrs, CT 06269, USA

e-mail: gordina@math.uconn.edu 


\section{Contents}

1 Introduction . . . . . . . . . . . . . . . . . . . . . . . . . 482

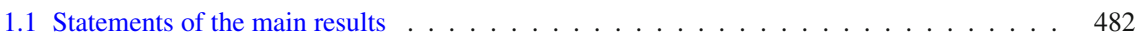

1.2 Discussion . . . . . . . . . . . . . . . . . . . . . . . . 486

2 Complex abstract Wiener spaces . . . . . . . . . . . . . . . . . . . . . . . . . . . . . 487

2.1 The structure of the projections . . . . . . . . . . . . . . . . . . . . . . . 492

3 Complex Heisenberg like groups . . . . . . . . . . . . . . . . . . . . . . . . . . . . . . 492

4 Brownian motion and heat kernel measures . . . . . . . . . . . . . . . . . . . . . . . . . . . . 494

4.1 Heat kernel quasi-invariance properties . . . . . . . . . . . . . . . . . . . . . . . 496

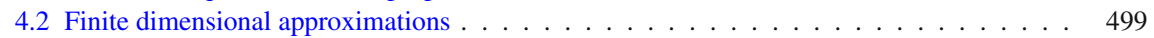

5 Holomorphic functions on $G$ and $G_{C M} \ldots \ldots \ldots \ldots \ldots \ldots$. . . . . . . . . . . . . 500

5.1 Holomorphic functions on Banach spaces . . . . . . . . . . . . . . . . . . 500

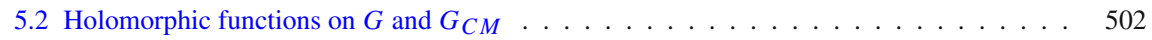

6 The Taylor isomorphism theorem . . . . . . . . . . . . . . . . . . . . . 506

6.1 A non-commutative Fock space . . . . . . . . . . . . . . . . . . . 506

6.2 The Taylor isomorphism . . . . . . . . . . . . . . . . . . . . 508

7 Density theorems . . . . . . . . . . . . . . . . . . . . . . . . . . . . . . . . . . . . . . . . . . . . .

7.1 Finite rank subspaces . . . . . . . . . . . . . . . . . . . . . . 512

7.2 Polynomial approximations . . . . . . . . . . . . . . . . . . . 515

8 The skeleton isomorphism . . . . . . . . . . . . . . . . . . . . . . 520

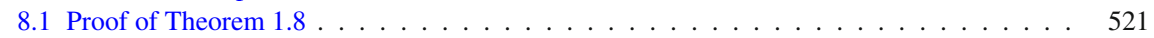

9 The holomorphic chaos expansion . . . . . . . . . . . . . . . . . . . . . 522

9.1 Generalities about multiple Itô integrals . . . . . . . . . . . . . . . . . . . . . . . 522

9.2 The stochastic Taylor map . . . . . . . . . . . . . . . . . . . . . . . . 525

10 Future directions and questions . . . . . . . . . . . . . . . . . . . . . . . 526

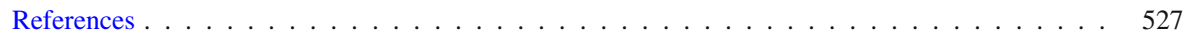

\section{Introduction}

The aim of this paper is to study spaces of holomorphic functions on an infinitedimensional Heisenberg like group based on a complex abstract Wiener space. In particular, we prove Taylor, skeleton, and holomorphic chaos isomorphism theorems. The tools we use come from properties of heat kernel measures on such groups which have been constructed and studied in [4]. We will state the main results of our paper and then conclude this introduction with a brief discussion of how our results relate to the existing literature.

\subsection{Statements of the main results}

\subsubsection{The Heisenberg like groups and heat kernel measures}

The basic input to our theory is a complex abstract Wiener space, $(W, H, \mu)$, as in Notation 2.4 which is equipped with a continuous skew-symmetric bi-linear form $\omega: W \times W \rightarrow \mathbf{C}$ as in Notation 3.1. Here and throughout this paper, $\mathbf{C}$ is a finite dimensional complex inner product space. The space, $G:=W \times \mathbf{C}$, becomes an infinite-dimensional "Heisenberg like" group when equipped with the following mul- 
tiplication rule

$$
\left(w_{1}, c_{1}\right) \cdot\left(w_{2}, c_{2}\right)=\left(w_{1}+w_{2}, c_{1}+c_{2}+\frac{1}{2} \omega\left(w_{1}, w_{2}\right)\right) \text {. }
$$

A typical example of such a group is the Heisenberg group of a symplectic vector space, but in our setting we have an additional structure of an abstract Wiener space to carry out the heat kernel measure analysis.

The group $G$ contains the Cameron-Martin group, $G_{C M}:=H \times \mathbf{C}$, as a subgroup. The Lie algebras of $G$ and $G_{C M}$ will be denoted by $\mathfrak{g}$ and $\mathfrak{g}_{C M}$ respectively which, as sets, may be identified with $G$ and $G_{C M}$ respectively-see Definition 3.2, Notation 3.3, and Proposition 3.5 for more details.

Let $b(t)=\left(B(t), B_{0}(t)\right)$ be a Brownian motion on $\mathfrak{g}$ associated to the natural Hilbertian structure on $\mathfrak{g}_{C M}$ as described in Eq. (4.1). The Brownian motion $\{g(t)\}_{t \geq 0}$ on $G$ is then the solution to the stochastic differential equation,

$$
d g(t)=g(t) \circ d b(t) \text { with } g(0)=\mathbf{e}=(0,0) .
$$

The explicit solution to Eq. (1.2) may be found in Eq. (4.2). For each $T>0$ we let $v_{T}:=\operatorname{Law}(g(T))$ be the heat kernel measure on $G$ at time $T$ as explained in Definitions 4.1 and 4.2. Analogous to the abstract Wiener space setting, $v_{T}$ is left (right) quasi-invariant by an element, $h \in G$, iff $h \in G_{C M}$, while $v_{T}\left(G_{C M}\right)=0$, see Theorem 4.5, Proposition 4.6, and [4, Proposition 6.3].

In addition to the above infinite-dimensional structures we will need corresponding finite dimensional approximations. These approximations will be indexed by $\operatorname{Proj}(W)$ which we now define.

Notation 1.1 Let $\operatorname{Proj}(W)$ denote the collection of finite rank continuous linear maps, $P: W \rightarrow H$, such that $\left.P\right|_{H}$ is an orthogonal projection. (Explicitly, $P$ must be as in Eq. (2.17) below.) Further, let $G_{P}:=P W \times \mathbf{C}$ (a subgroup of $\left.G_{C M}\right)$ and $\pi_{P}: G \rightarrow G_{P}$ be the projection map defined by $\pi_{P}(w, c):=(P w, c)$.

To each $P \in \operatorname{Proj}(W), G_{P}$ is a finite dimensional Lie group. The Brownian motions and heat kernel measures, $\left\{v_{t}^{P}\right\}_{t>0}$, on $G_{P}$ are constructed similarly to those on $G$-see Definition 4.10. We will use $\left\{\left(G_{P}, v_{T}^{P}\right)\right\}_{P \in \operatorname{Proj}(W)}$ as finite dimensional approximations to $\left(G, v_{T}\right)$.

\subsubsection{The Taylor isomorphism theorem}

The Taylor map, $\mathcal{T}_{T}$, is a unitary map relating the "square integrable" holomorphic functions on $G_{C M}$ with the collection of their derivatives at $\mathbf{e} \in G_{C M}$. Before we can state this theorem we need to introduce the two Hilbert spaces involved.

In what follows, $\mathcal{H}\left(G_{C M}\right)$ and $\mathcal{H}(G)$ will denote the space of holomorphic functions on $G_{C M}$ and $G$ respectively. (See Sect. 5 for the properties of these function spaces which are used throughout this paper.) We also let $\mathbf{T}:=\mathbf{T}\left(\mathfrak{g}_{C M}\right)$ be the algebraic tensor algebra over $\mathfrak{g}_{C M}, \mathbf{T}^{\prime}$ be its algebraic dual, $J$ be the two-sided ideal in $\mathbf{T}$ 
generated by

$$
\left\{h \otimes k-k \otimes h-[h, k]: h, k \in \mathfrak{g}_{C M}\right\},
$$

and $J^{0}=\left\{\alpha \in \mathbf{T}^{\prime}: \alpha(J)=0\right\}$ be the backwards annihilator of $J$-see Notation 6.1. Given $f \in \mathcal{H}(G)$ we let $\alpha:=\mathcal{T} f$ denote the element of $J^{0}$ defined by $\langle\alpha, 1\rangle=f(\mathbf{e})$ and

$$
\left\langle\alpha, h_{1} \otimes \cdots \otimes h_{n}\right\rangle:=\left(\tilde{h}_{1} \ldots \tilde{h}_{n} f\right)(\mathbf{e})
$$

where $h_{i} \in \mathfrak{g}_{C M}$ and $\tilde{h}_{i}$ is the left invariant vector field on $G_{C M}$ agreeing with $h_{i}$ at e-see Proposition 3.5 and Definition 6.2. We call $\mathcal{T}$ the Taylor map since $\mathcal{T} f \in$ $J^{0}\left(\mathfrak{g}_{C M}\right)$ encodes all of the derivatives of $f$ at $\mathbf{e}$.

Definition 1.2 ( $L^{2}$-holomorphic functions on $\left.G_{C M}\right)$ For $T>0$, let

$$
\begin{gathered}
\|f\|_{\mathcal{H}_{T}^{2}\left(G_{C M}\right)}=\sup _{P \in \operatorname{Proj}(W)}\left\|\left.f\right|_{G_{P}}\right\|_{L^{2}\left(G_{P}, \nu_{T}^{P}\right)} \quad \text { for all } f \in \mathcal{H}\left(G_{C M}\right), \text { and } \\
\mathcal{H}_{T}^{2}\left(G_{C M}\right):=\left\{f \in \mathcal{H}\left(G_{C M}\right):\|f\|_{\mathcal{H}_{T}^{2}\left(G_{C M}\right)}<\infty\right\} .
\end{gathered}
$$

In Corollary 6.6 below, we will see that $\mathcal{H}_{T}^{2}\left(G_{C M}\right)$ is not empty and in fact contains the space of holomorphic cylinder polynomials $\left(\mathcal{P}_{C M}\right)$ on $G_{C M}$ described in Eq. (1.7) below. Despite the fact that $\nu_{T}\left(G_{C M}\right)=0, \mathcal{H}_{T}^{2}\left(G_{C M}\right)$ should roughly be thought of as the $v_{T}$-square integrable holomorphic functions on $G_{C M}$.

Definition 1.3 (Non-commutative Fock space) Let $T>0$ and

$$
\|\alpha\|_{J_{T}^{0}\left(\mathfrak{g}_{C M}\right)}^{2}:=\sum_{n=0}^{\infty} \frac{T^{n}}{n !} \sum_{h_{1}, \ldots, h_{n} \in S}\left|\left\langle\alpha, h_{1} \otimes \cdots \otimes h_{n}\right\rangle\right|^{2} \quad \text { for all } \alpha \in J^{0}\left(\mathfrak{g}_{C M}\right),
$$

where $S$ is any orthonormal basis for $\mathfrak{g}_{C M}$. The non-commutative Fock space is defined as

$$
J_{T}^{0}\left(\mathfrak{g}_{C M}\right):=\left\{\alpha \in J^{0}\left(\mathfrak{g}_{C M}\right):\|\alpha\|_{J_{T}^{0}\left(\mathfrak{g}_{C M}\right)}^{2}<\infty\right\} .
$$

It is easy to see that $\|\cdot\|_{J_{T}^{0}\left(\mathfrak{g}_{C M}\right)}$ is a Hilbertian norm on $J_{T}^{0}\left(\mathfrak{g}_{C M}\right)$-see Definition 6.4 and Eq. (6.8). For a detailed introduction to such Fock spaces we refer to [13].

Remark 1.4 When $\omega=0, G(\omega)$ is commutative and the Fock space, $J_{T}^{0}\left(\mathfrak{g}_{C M}\right)$, becomes the standard commutative bosonic Fock space of symmetric tensors over $\mathfrak{g}_{C M}^{*}$.

The following theorem is proved in Sect. 6-see Theorem 6.10.

Theorem 1.5 (The Taylor isomorphism) For all $T>0, \mathcal{T}\left(\mathcal{H}_{T}^{2}\left(G_{C M}\right)\right) \subset J_{T}^{0}\left(\mathfrak{g}_{C M}\right)$ and the linear map,

$$
\mathcal{T}_{T}:=\left.\mathcal{T}\right|_{\mathcal{H}_{T}^{2}\left(G_{C M}\right)}: \mathcal{H}_{T}^{2}\left(G_{C M}\right) \rightarrow J_{T}^{0}\left(\mathfrak{g}_{C M}\right)
$$

is unitary. 
Associated to this theorem is an analogue of Bargmann's pointwise bounds which appear in Theorem 6.11 below.

\subsubsection{The skeleton isomorphism theorem}

Similarly to how it has been done on a complex abstract Wiener space by Sugita $[26,27]$, the quasi-invariance of the heat kernel measure $v_{T}$ allows us to define the skeleton map from $L^{p}\left(G, v_{T}\right)$ to a space of functions on the Cameron-Martin subgroup $G_{C M}$, a set of $\nu_{T}$-measure 0 .

Definition 1.6 A holomorphic cylinder polynomial on $G$ is a holomorphic cylinder function (see Definition 4.3) of the form, $f=F \circ \pi_{P}: G \rightarrow \mathbb{C}$, where $P \in \operatorname{Proj}(W)$ and $F: P W \times \mathbf{C} \rightarrow \mathbb{C}$ is a holomorphic polynomial. The space of holomorphic cylinder polynomials will be denoted by $\mathcal{P}$.

The "Gaussian" heat kernel bounds in Theorem 4.11 easily imply that $\mathcal{P} \subset L^{p}\left(v_{T}\right)$ for all $p<\infty$-see Corollary 5.10.

Definition 1.7 (Holomorphic $L^{p}$-functions) For $T>0$ and $1 \leqslant p<\infty$, let $\mathcal{H}_{T}^{p}(G)$ denote the $L^{p}\left(v_{T}\right)$-closure of $\mathcal{P} \subset L^{p}\left(v_{T}\right)$.

From Corollary 4.8 below, if $T>0, p \in(1, \infty], f \in L^{p}\left(G, v_{T}\right)$, and $h \in G_{C M}$, then $\int_{G}|f(h \cdot g)| d \nu_{T}(g)<\infty$. Thus, if $f \in \mathcal{H}_{T}^{2}(G)$ we may define the skeleton map (see Definition 4.7) by

$$
\left(S_{T} f\right)(h):=\int_{G} f(h \cdot g) d v_{T}(g) .
$$

It is shown in Theorem 5.12 that $S_{T}\left(\mathcal{H}_{T}^{2}(G)\right) \subset \mathcal{H}_{T}^{2}\left(G_{C M}\right)$ for all $T>0$.

Theorem 1.8 (The skeleton isomorphism) For each $T>0$, the skeleton map, $S_{T}: \mathcal{H}_{T}^{2}(G) \rightarrow \mathcal{H}_{T}^{2}\left(G_{C M}\right)$, is unitary.

Following Sugita's results [26,27] in the case of an abstract Wiener space, we call $\left.S_{T}\right|_{\mathcal{H}_{T}^{2}(G)}$ the skeleton map since it characterizes $f \in \mathcal{H}_{T}^{2}(G)$ by its "values", $S_{T} f$, on $G_{C M}$. Sugita would refer to $G_{C M}$ as the skeleton of $G(\omega)$ owing to the fact that $v_{T}\left(G_{C M}\right)=0$ as we show in Proposition 4.6.

Theorem 1.8 is proved in Sect. 8 and relies on two key density results from Sect. 7. The first is Lemma 7.3 (an infinite-dimensional version of [7, Lemma 3.5]) which states that the finite rank tensors (see Definition 7.2) are dense inside of $J_{T}^{0}\left(\mathfrak{g}_{C M}\right)$. The second is Theorem 7.1 which states that

$$
\mathcal{P}_{C M}:=\left\{\left.p\right|_{G_{C M}}: p \in \mathcal{P}\right\}
$$

is a dense subspace of $\mathcal{H}_{T}^{2}\left(G_{C M}\right)$. Matt Cecil [2] has modified the arguments presented in Sect. 7 to cover the situation of path groups over graded nilpotent Lie groups. Cecil's arguments are necessarily much more involved because his Lie groups have nilpotency of arbitrary step. 


\subsubsection{The holomorphic chaos expansion}

So far we have produced (for each $T>0$ ) two unitary isomorphisms, the skeleton map $S_{T}$ and the Taylor isomorphism $\mathcal{T}_{T}$,

$$
\mathcal{H}_{T}^{2}(G) \underset{S_{T}}{\cong} \mathcal{H}_{T}^{2}\left(G_{C M}\right) \underset{\stackrel{\mathcal{T}_{T}}{\longrightarrow}}{\cong} J_{T}^{0}\left(\mathfrak{g}_{C M}\right)
$$

The next theorem gives an explicit formula for $\left(\mathcal{T}_{T} \circ S_{T}\right)^{-1}: J_{T}^{0}\left(\mathfrak{g}_{C M}\right) \rightarrow \mathcal{H}_{T}^{2}(G)$.

Theorem 1.9 (The holomorphic chaos expansion) If $f \in \mathcal{H}_{T}^{2}(G)$ and $\alpha_{f}:=\mathcal{T}_{T} S_{T} f$, then

$$
f(g(T))=\sum_{n=0}^{\infty}\left\langle\alpha_{f}, \int_{0 \leq s_{1} \leq s_{2} \leq \cdots \leq s_{n} \leq T} d b\left(s_{1}\right) \otimes \cdots \otimes d b\left(s_{n}\right)\right\rangle
$$

where $b(t)$ and $g(t)$ are related as in Eq. (1.2) or equivalently as in Eq. (4.2).

This result is proved in Sect. 9 and in particular, see Theorem 9.10. The precise meaning of the right hand side of Eq. (1.8) is also described there.

\subsection{Discussion}

As we noticed in Remark 1.4 when the form $\omega \equiv 0$ the Fock space $J_{T}^{0}\left(\mathfrak{g}_{C M}\right)$ is the standard commutative bosonic Fock space [9]. In this case the Taylor map is one of three isomorphisms between different representations of a Fock space, one other being the Segal-Bargmann transform. The history of the latter is described in [13] beginning with works of Bargmann [1] and Segal [24]. For other relevant results see [8,14].

To put our results into perspective, recall that the classical Segal-Bargmann space is the Hilbert space of holomorphic functions on $\mathbb{C}^{n}$ that are square-integrable with respect to the Gaussian measure $d \mu_{n}(z)=\pi^{-n} e^{-|z|^{2}} d z$, where $d z$ is the $2 n$-dimensional Lebesgue measure. One of the features of functions in the Segal-Bargmann space is that they satisfy the pointwise bounds $|f(z)| \leqslant\|f\|_{L^{2}\left(\mu_{n}\right)} \exp \left(|z|^{2} / 2\right)$ (compare with Theorem 6.11). As it is described in [13], if $\mathbb{C}^{n}$ is replaced by an infinite-dimensional complex Hilbert space $H$, one of the first difficulties is to find a suitable version of the Gaussian measure. It can be achieved, but only on a certain extension $W$ of $H$, which leads one to consider the complex abstract Wiener space setting. From Sugita's [26,27] work on holomorphic functions over a complex abstract Wiener space, it is known that the pointwise bounds control only the values of the holomorphic functions on $H$. This difficulty explains, in part, the need to consider two function spaces: one is of holomorphic functions on $H$ (or $G_{C M}$ in our case) versus the square-integrable (weakly) holomorphic functions on $W$ (or $G$ in our case).

The Taylor map has also been studied in other non-commutative infinitedimensional settings. Gordina [10-12] considered the Taylor isomorphism in the context of Hilbert-Schmidt groups, while Cecil [2] considered the Taylor isomorphism for path groups over stratified Lie groups. The nilpotentcy of the Heisenberg 
like groups studied in this paper allow us to give a more complete description of the square integrable holomorphic function spaces than was possible in [10-12] for the Hilbert-Schmidt groups.

Complex analysis in infinite dimensions in a somewhat different setting has been studied by Lempert (e.g.[20]), and for more results on Gaussian-like measures on infinite-dimensional curved spaces see papers by Pickrell (e.g.[22,23]). For another view of different representations of Fock space, one can look at results in the field of white noise, as presented in the book by Obata [21]. The map between an $L^{2}$-space and a space of symmetric tensors sometimes is called the Segal isomorphism as in $[18,19]$. For more background on this and related topics see [16].

\section{Complex abstract Wiener spaces}

Suppose that $W$ is a complex separable Banach space and $\mathcal{B}_{W}$ is the Borel $\sigma$-algebra on $W$. Let $W_{\mathrm{Re}}$ denote $W$ thought of as a real Banach space. For $\lambda \in \mathbb{C}$, let $M_{\lambda}: W \rightarrow W$ be the operation of multiplication by $\lambda$.

Definition 2.1 A measure $\mu$ on $\left(W, \mathcal{B}_{W}\right)$ is called a (mean zero, non-degenerate) Gaussian measure provided that its characteristic functional is given by

$$
\hat{\mu}(u):=\int_{W} e^{i u(w)} d \mu(w)=e^{-\frac{1}{2} q(u, u)} \quad \text { for all } u \in W_{\mathrm{Re}}^{*},
$$

where $q=q_{\mu}: W_{\mathrm{Re}}^{*} \times W_{\mathrm{Re}}^{*} \rightarrow \mathbb{R}$ is an inner product on $W_{\mathrm{Re}}^{*}$. If in addition, $\mu$ is invariant under multiplication by $i$, that is, $\mu \circ M_{i}^{-1}=\mu$, we say that $\mu$ is a complex Gaussian measure on $W$.

Remark 2.2 Suppose $W=\mathbb{C}^{d}$ and let us write $w \in W$ as $w=x+i y$ with $x, y \in \mathbb{R}^{d}$. Then the most general Gaussian measure on $W$ is of the form

$$
d \mu(w)=\frac{1}{Z} \exp \left(-\frac{1}{2} Q\left[\begin{array}{l}
x \\
y
\end{array}\right] \cdot\left[\begin{array}{l}
x \\
y
\end{array}\right]\right) d x d y
$$

where $Q$ is a real positive definite $2 d \times 2 d$ matrix and $Z$ is a normalization constant. The matrix $Q$ may be written in $2 \times 2$ block form as

$$
Q=\left[\begin{array}{cc}
A & B \\
B^{\operatorname{tr}} & C
\end{array}\right]
$$

A simple exercise shows $\mu=\mu \circ M_{i}^{-1}$ iff $B=0$ and $A=C$. Thus the general complex Gaussian measure on $\mathbb{C}^{d}$ is of the form 


$$
\begin{aligned}
d \mu(w) & =\frac{1}{Z} \exp \left(-\frac{1}{2}(A x \cdot x+A y \cdot y)\right) d x d y \\
& =\frac{1}{Z} \exp \left(-\frac{1}{2} A w \cdot \bar{w}\right) d x d y
\end{aligned}
$$

where $A$ is a real positive definite matrix.

Given a complex Gaussian measure $\mu$ as in Definition 2.1, let

$$
\|w\|_{H}:=\sup _{u \in W_{\operatorname{Re}}^{*} \backslash\{0\}} \frac{|u(w)|}{\sqrt{q(u, u)}} \quad \text { for all } w \in W,
$$

and define the Cameron-Martin subspace, $H \subset W$, by

$$
H=\left\{h \in W:\|h\|_{H}<\infty\right\}
$$

The following theorem summarizes some of the standard properties of the triple $(W, H, \mu)$.

Theorem 2.3 Let $(W, H, \mu)$ be as above, where $\mu$ is a complex Gaussian measure on $\left(W, \mathcal{B}_{W}\right)$. Then

(1) $H$ is a dense complex subspace of $W$.

(2) There exists a unique inner product, $\langle\cdot, \cdot\rangle_{H}$, on $H$ such that $\|h\|_{H}^{2}=\langle h, h\rangle$ for all $h \in H$. Moreover, with this inner product $H$ is a complete separable complex Hilbert space.

(3) There exists $C<\infty$ such that

$$
\|h\|_{W} \leqslant C\|h\|_{H} \quad \text { for any } h \in H .
$$

(4) If $\left\{e_{j}\right\}_{j=1}^{\infty}$ is an orthonormal basis for $H$ and $u, v \in W_{\mathrm{Re}}^{*}$, then

$$
q(u, v)=\langle u, v\rangle_{H_{\mathrm{Re}}^{*}}=\sum_{j=1}^{\infty}\left[u\left(e_{j}\right) v\left(e_{j}\right)+u\left(i e_{j}\right) v\left(i e_{j}\right)\right]
$$

(5) $\mu \circ M_{\lambda}^{-1}=\mu$ for all $\lambda \in \mathbb{C}$ with $|\lambda|=1$.

Proof We will begin with the proof of item 5. From Eq. (2.1), the invariance of $\mu$ under multiplication by $i\left(\mu \circ M_{i}^{-1}=\mu\right)$ is equivalent to assuming that $q\left(u \circ M_{i}\right.$, $\left.u \circ M_{i}\right)=q(u, u)$ for all $u \in W_{\operatorname{Re}}^{*}$. By polarization, we may further conclude that

$$
q\left(u \circ M_{i}, v \circ M_{i}\right)=q(u, v) \quad \text { for all } u, v \in W_{\mathrm{Re}}^{*} .
$$

Taking $v=u \circ M_{i}$ in this identity then shows that $q\left(u \circ M_{i},-u\right)=q\left(u, u \circ M_{i}\right)$ and hence that

$$
q\left(u, u \circ M_{i}\right)=0 \quad \text { for any } u \in W_{\mathrm{Re}}^{*} .
$$


Therefore if $\lambda=a+i b$ with $a, b \in \mathbb{R}$, we see that

$$
\begin{aligned}
q\left(u \circ M_{\lambda}, u \circ M_{\lambda}\right) & =q\left(a u+b u \circ M_{i}, a u+b u \circ M_{i}\right) \\
& =\left(a^{2}+b^{2}\right) q(u, u)=|\lambda|^{2} q(u, u),
\end{aligned}
$$

from which it follows that $q\left(u \circ M_{\lambda}, u \circ M_{\lambda}\right)=q(u, u)$ for all $u \in W_{\operatorname{Re}}^{*}$ and $|\lambda|=1$. Coupling this observation with Eq. (2.1) implies $\mu \circ M_{\lambda}^{-1}=\mu$ for all $|\lambda|=1$. If $|\lambda|=1$, from Eqs. (2.2) and (2.8), it follows that

$$
\begin{aligned}
\|\lambda w\|_{H} & =\sup _{u \in W_{\mathrm{Re}}^{*} \backslash\{0\}} \frac{|u(\lambda w)|}{\sqrt{q(u, u)}}=\sup _{u \in W_{\mathrm{Re}}^{*} \backslash\{0\}} \frac{\left|u \circ M_{\lambda}(w)\right|}{\sqrt{q\left(u \circ M_{\lambda}, u \circ M_{\lambda}\right)}} \\
& =\sup _{u \in W_{\mathrm{Re}}^{*} \backslash\{0\}} \frac{|u(w)|}{\sqrt{q(u, u)}}=\|w\|_{H} \text { for all } w \in W .
\end{aligned}
$$

In particular, if $\|h\|_{H}<\infty$ and $|\lambda|=1$, then $\|\lambda h\|_{H}=\|h\|_{H}<\infty$ and hence $\lambda H \subset H$ which shows that $H$ is a complex subspace of $W$. From [4, Theorem 2.3] summarizing some well-known properties of Gaussian measures, we know that item 3. holds, $H$ is a dense subspace of $W_{\mathrm{Re}}$, and there exists a unique real Hilbertian inner product, $\langle\cdot, \cdot\rangle_{H_{\mathrm{Re}}}$, on $H$ such that $\|h\|_{H}^{2}=\langle h, h\rangle_{H_{\mathrm{Re}}}$ for all $h \in H$. Polarizing the identity $\|\lambda h\|_{H}=\|h\|_{H}$ implies $\langle\lambda h, \lambda k\rangle_{H_{\mathrm{Re}}}=\langle h, k\rangle_{H_{\mathrm{Re}}}$ for all $h, k \in H$. Taking $\lambda=i$ and $k=-i h$ then shows $\langle i h, h\rangle_{\operatorname{Re}}=\langle h,-i h\rangle_{\operatorname{Re}}$, and hence that $\langle i h, h\rangle_{\operatorname{Re}}=0$ for all $h \in H$. Using this information it is a simple matter to check that

$$
\langle h, k\rangle_{H}:=\langle h, k\rangle_{H_{\mathrm{Re}}}+i\langle h, i k\rangle_{H_{\mathrm{Re}}} \quad \text { for all } h, k \in H,
$$

is the unique complex inner product on $H$ such that $\operatorname{Re}\langle\cdot, \cdot\rangle_{H}=\langle\cdot, \cdot\rangle_{H_{\mathrm{Re}}}$.

So it only remains to prove Eq. (2.5). For a proof of the first equality in Eq. (2.5), see [4, Theorem 2.3]. To prove the second equality in this equation, it suffices to observe that $\left\{e_{j}, i e_{j}\right\}_{j=1}^{\infty}$ is an orthonormal basis for $\left(H_{\mathrm{Re}},\langle\cdot, \cdot\rangle_{H_{\mathrm{Re}}}\right)$ and therefore,

$$
\langle u, v\rangle_{H_{\mathrm{Re}}^{*}}=\sum_{j=1}^{\infty}\left[u\left(e_{j}\right) v\left(e_{j}\right)+u\left(i e_{j}\right) v\left(i e_{j}\right)\right] \quad \text { for any } u, v \in H_{\mathrm{Re}}^{*}
$$

Notation 2.4 The triple, $(W, H, \mu)$, appearing in Theorem 2.3 will be called a complex abstract Wiener space (Notice that there is redundancy in this notation since $\mu$ is determined by $H$, and $H$ is determined by $\mu$ ).

Lemma 2.5 Suppose that $u, v \in W_{\mathrm{Re}}^{*}$ and $a, b \in \mathbb{C}$, then

$$
\int_{W} e^{a u+b v} d \mu=\exp \left(\frac{1}{2}\left(a^{2} q(u, u)+b^{2} q(v, v)+2 a b q(u, v)\right)\right) .
$$


Proof Equation (2.10) is easily verified when both $a$ and $b$ are real. This suffices to complete the proof, since both sides of Eq. (2.10) are analytic functions of $a, b \in \mathbb{C}$.

Lemma 2.6 Let $(W, H, \mu)$ be a complex abstract Wiener space, then for any $\varphi \in W^{*}$, we have

$$
\begin{aligned}
& \int_{W} e^{\varphi(w)} d \mu(w)=1=\int_{W} e^{\overline{\varphi(w)}} d \mu(w), \\
& \int_{W}|\operatorname{Re} \varphi(w)|^{2} d \mu(w)=\int_{W}|\operatorname{Im} \varphi(w)|^{2} d \mu(w)=\|\varphi\|_{H^{*}}^{2},
\end{aligned}
$$

and

$$
\int_{W}|\varphi(w)|^{2} d \mu(w)=2\|\varphi\|_{H^{*}}^{2} .
$$

More generally, if $\mathbf{C}$ is another complex Hilbert space and $\varphi \in L(W, \mathbf{C})$, then

$$
\int_{W}\|\varphi(w)\|_{\mathbf{C}}^{2} d \mu(w)=2\|\varphi\|_{H^{*} \otimes \mathbf{C}}^{2} \cdot
$$

Proof If $u=\operatorname{Re} \varphi$, then $\varphi(w)=u(w)-i u(i w)$. Therefore by Eqs. (2.6), (2.7), and (2.10),

$$
\begin{aligned}
\int_{W} e^{\varphi} d \mu & =\int_{W} e^{u-i u \circ M_{i}} d \mu \\
& =\exp \left(\frac{1}{2}\left(q(u, u)-q\left(u \circ M_{i}, u \circ M_{i}\right)-2 i q\left(u, u \circ M_{i}\right)\right)\right)=1 .
\end{aligned}
$$

Taking the complex conjugation of this identity shows $\int_{W} e^{\overline{\varphi(w)}} d \mu(w)=1$. Also using Lemma 2.5, we have

$$
\begin{aligned}
& \int_{W}|\operatorname{Re} \varphi(w)|^{2} d \mu(w)=q(u, u) \text { and } \\
& \int_{W}|\operatorname{Im} \varphi(w)|^{2} d \mu(w)=\int_{W}|u(i w)|^{2} d \mu(w)=q\left(u \circ M_{i}, u \circ M_{i}\right)=q(u, u) .
\end{aligned}
$$


To evaluate $q(u, u)$, let $\left\{e_{k}\right\}_{k=1}^{\infty}$ be an orthonormal basis for $H$ so that $\left\{e_{k}, i e_{k}\right\}_{k=1}^{\infty}$ is an orthonormal basis for $\left(H_{\operatorname{Re}}, \operatorname{Re}\langle\cdot, \cdot\rangle_{H}\right)$. Then by Eq. (2.5),

$$
q(u, u)=\sum_{k=1}^{\infty}\left[\left|u\left(e_{k}\right)\right|^{2}+\left|u\left(i e_{k}\right)\right|^{2}\right]=\sum_{k=1}^{\infty}\left|\varphi\left(e_{k}\right)\right|^{2}=\|\varphi\|_{H^{*}}^{2}
$$

To prove Eq. (2.14), apply [4, Eq. (2.13)] to find

$$
\begin{aligned}
\int_{W}\|\varphi(w)\|_{\mathbf{C}}^{2} d \mu(w) & =\sum_{k=1}^{\infty}\left[\left\|\varphi\left(e_{k}\right)\right\|_{\mathbf{C}}^{2}+\left\|\varphi\left(i e_{k}\right)\right\|_{\mathbf{C}}^{2}\right] \\
& =2 \sum_{k=1}^{\infty}\left\|\varphi\left(e_{k}\right)\right\|_{\mathbf{C}}^{2}=2\|\varphi\|_{H^{*} \otimes \mathbf{C}}^{2}
\end{aligned}
$$

Remark 2.7 (Heat kernel interpretation of Lemma 2.6) The measure $\mu$ formally satisfies

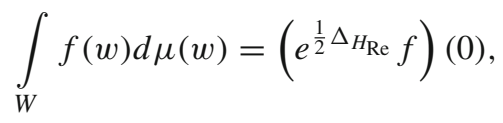

where $\Delta_{H_{\operatorname{Re}}}=\sum_{j=1}^{\infty} \partial_{e_{j}}^{2}$ and $\left\{e_{j}\right\}_{j=1}^{\infty}$ is an orthonormal basis for $H_{\mathrm{Re}}$. If $f$ is holomorphic or anti-holomorphic, then $f$ is harmonic and therefore

$$
\int_{W} f(w) d \mu(w)=\left(e^{\frac{1}{2} \Delta_{H_{\mathrm{Re}}}} f\right)(0)=f(0) .
$$

Applying this identity to $f(w)=e^{\varphi(w)}$ or $f(w)=\overline{e^{\varphi(w)}}$ with $\varphi \in W^{*}$ gives Eq. (2.11). If $u \in W_{\mathrm{Re}}^{*}$, we have

$$
\begin{aligned}
& \int_{W} u^{2}(w) d \mu(w)=\left(e^{\frac{1}{2} \Delta_{H_{\mathrm{Re}}}} u^{2}\right)(0)=\sum_{n=0}^{\infty} \frac{1}{2^{n} n !}\left(\Delta_{H_{\mathrm{Re}}}^{n} u^{2}\right)(0) \\
& =\frac{1}{2}\left(\Delta_{H_{\mathrm{Re}}} u^{2}\right)(0)=\frac{1}{2} \sum_{j=1}^{\infty}\left(\partial_{e_{j}}^{2} u^{2}\right)(0) \\
& =\sum_{j=1}^{\infty} u\left(e_{j}\right)^{2}=\|u\|_{H_{\mathrm{Re}}}^{2} \text {. }
\end{aligned}
$$

Eqs. (2.12) and (2.13) now follow easily from this identity. 


\subsection{The structure of the projections}

Let $i: H \rightarrow W$ be the inclusion map and $i^{*}: W^{*} \rightarrow H^{*}$ be its transpose, i.e. $i^{*} \ell:=\ell \circ i$ for all $\ell \in W^{*}$. Also let

$$
H_{*}:=\left\{h \in H:\langle\cdot, h\rangle_{H} \in \operatorname{Ran}\left(i^{*}\right) \subset H^{*}\right\}
$$

or in other words, $h \in H$ is in $H_{*}$ iff $\langle\cdot, h\rangle_{H} \in H^{*}$ extends to a continuous linear functional on $W$. (We will continue to denote the continuous extension of $\langle\cdot, h\rangle_{H}$ to $W$ by $\langle\cdot, h\rangle_{H}$.) Because $H$ is a dense subspace of $W, i^{*}$ is injective, and because $i$ is injective, $i^{*}$ has a dense range. Since $h \in H \rightarrow\langle\cdot, h\rangle_{H} \in H^{*}$ is a conjugate linear isometric isomorphism, it follows from the above comments that $H_{*} \ni h \rightarrow\langle\cdot, h\rangle_{H} \in W^{*}$ is a conjugate linear isomorphism too, and that $H_{*}$ is a dense subspace of $H$.

Lemma 2.8 There is a one to one correspondence between Proj( $W$ ) (see Notation 1.1) and the collection of finite rank orthogonal projections, $P$, on $H$ such that $P H \subset H_{*}$.

Proof If $P \in \operatorname{Proj}(W)$ and $u \in P W \subset H$, then, because $\left.P\right|_{H}$ is an orthogonal projection, we have

$$
\langle P h, u\rangle_{H}=\langle h, P u\rangle_{H}=\langle h, u\rangle_{H} \quad \text { for all } h \in H .
$$

Since $P: W \rightarrow H$ is continuous, it follows that $u \in H_{*}$, i.e. $P W \subset H_{*}$.

Conversely, suppose that $P: H \rightarrow H$ is a finite rank orthogonal projection such that $P H \subset H_{*}$. Let $\left\{e_{j}\right\}_{j=1}^{n}$ be an orthonormal basis for $P H$ and $\ell_{j} \in W^{*}$ such that $\left.\ell_{j}\right|_{H}=\left\langle\cdot, e_{j}\right\rangle_{H}$. Then we may extend $P$ uniquely to a continuous operator from $W$ to $H$ (still denoted by $P$ ) by letting

$$
P w:=\sum_{j=1}^{n} \ell_{j}(w) e_{j}=\sum_{j=1}^{n}\left\langle w, e_{j}\right\rangle_{H} e_{j} \quad \text { for all } w \in W
$$

From [4, Eq. 3.43], there exists $C=C(P)<\infty$ such that

$$
\|P w\|_{H} \leqslant C\|w\|_{W} \text { for all } w \in W .
$$

\section{Complex Heisenberg like groups}

In this section we review the infinite-dimensional Heisenberg like groups and Lie algebras which were introduced in [4, Section 3].

Notation 3.1 Let $(W, H, \mu)$ be a complex abstract Wiener space, C be a complex finite dimensional inner product space, and $\omega: W \times W \rightarrow \mathbf{C}$ be a continuous skew symmetric bilinear quadratic form on $W$. Further, let

$$
\|\omega\|_{0}:=\sup \left\{\left\|\omega\left(w_{1}, w_{2}\right)\right\|_{\mathbf{C}}: w_{1}, w_{2} \in W \text { with }\left\|w_{1}\right\|_{W}=\left\|w_{2}\right\|_{W}=1\right\}
$$


be the uniform norm on $\omega$ which is finite by the assumed continuity of $\omega$.

Definition 3.2 Let $\mathfrak{g}$ denote $W \times \mathbf{C}$ when thought of as a Lie algebra with the Lie bracket operation given by

$$
[(A, a),(B, b)]:=(0, \omega(A, B))
$$

Let $G=G(\omega)$ denote $W \times \mathbf{C}$ when thought of as a group with the multiplication law given by

$$
g_{1} g_{2}=g_{1}+g_{2}+\frac{1}{2}\left[g_{1}, g_{2}\right] \quad \text { for any } g_{1}, g_{2} \in G
$$

or equivalently by Eq. (1.1).

It is easily verified that $\mathfrak{g}$ is a Lie algebra and $G$ is a group. The identity of $G$ is the zero element, $\mathbf{e}:=(0,0)$.

Notation 3.3 Let $\mathfrak{g}_{C M}$ denote $H \times \mathbf{C}$ when viewed as a Lie subalgebra of $\mathfrak{g}$ and $G_{C M}$ denote $H \times \mathbf{C}$ when viewed as a subgroup of $G=G(\omega)$. We will refer to $\mathfrak{g}_{C M}\left(G_{C M}\right)$ as the Cameron-Martin subalgebra (subgroup) of $\mathfrak{g}(G)$. (For explicit examples of such $(W, H, \mathbf{C}, \omega)$, see [4].)

We equip $G=\mathfrak{g}=W \times \mathbf{C}$ with the Banach space norm

$$
\|(w, c)\|_{\mathfrak{g}}:=\|w\|_{W}+\|c\|_{\mathbf{C}}
$$

and $G_{C M}=\mathfrak{g}_{C M}=H \times \mathbf{C}$ with the Hilbert space inner product,

$$
\langle(A, a),(B, b)\rangle_{\mathfrak{g}_{C M}}:=\langle A, B\rangle_{H}+\langle a, b\rangle_{\mathbf{C}}
$$

The associate Hilbertian norm is given by

$$
\|(A, a)\|_{\mathfrak{g}_{C M}}:=\sqrt{\|A\|_{H}^{2}+\|a\|_{\mathbf{C}}^{2}} .
$$

As was shown in [4, Lemma 3.3], these Banach space topologies on $W \times \mathbf{C}$ and $H \times \mathbf{C}$ make $G$ and $G_{C M}$ into topological groups.

Notation 3.4 (Linear differentials) Suppose $f: G \rightarrow \mathbb{C}$, is a Frechét smooth function. For $g \in G$ and $h, k \in \mathfrak{g}$ let

$$
f^{\prime}(g) h:=\partial_{h} f(g)=\left.\frac{d}{d t}\right|_{0} f(g+t h)
$$

and

$$
f^{\prime \prime}(g)(h \otimes k):=\partial_{h} \partial_{k} f(g)
$$

(Here and in the sequel a prime on a symbol will be used to denote its derivative or differential.) 
As $G$ itself is a vector space, the tangent space, $T_{g} G$, to $G$ at $g$ is naturally isomorphic to $G$. Indeed, if $v, g \in G$, then we may define a tangent vector $v_{g} \in T_{g} G$ by $v_{g} f=f^{\prime}(g) v$ for all Frechét smooth functions $f: G \rightarrow \mathbb{C}$. We will identify $\mathfrak{g}$ with $T_{\mathbf{e}} G$ and $\mathfrak{g}_{C M}$ with $T_{\mathbf{e}} G_{C M}$. Recall that as sets $\mathfrak{g}=G$ and $\mathfrak{g}_{C M}=G_{C M}$. For $g \in G$, let $l_{g}: G \rightarrow G$ be the left translation by $g$. For $h \in \mathfrak{g}$, let $\tilde{h}$ be the left invariant vector field on $G$ such that $\tilde{h}(g)=h$ when $g=\mathbf{e}$. More precisely, if $\sigma(t) \in G$ is any smooth curve such that $\sigma(0)=\mathbf{e}$ and $\dot{\sigma}(0)=h($ e.g. $\sigma(t)=t h)$, then

$$
\tilde{h}(g)=, l_{g *} h:=\left.\frac{d}{d t}\right|_{0} g \cdot \sigma(t)
$$

As usual, we view $\tilde{h}$ as a first order differential operator acting on smooth functions, $f: G \rightarrow \mathbb{C}$, by

$$
(\tilde{h} f)(g)=\left.\frac{d}{d t}\right|_{0} f(g \cdot \sigma(t))
$$

The proof of the following easy proposition may be found in [4, Proposition 3.7].

Proposition 3.5 Let $f: G \rightarrow \mathbb{C}$ be a smooth function, $h=(A, a) \in \mathfrak{g}$ and $g=$ $(w, c) \in G$. Then

$$
\widetilde{h}(g):=l_{g *} h=\left(A, a+\frac{1}{2} \omega(w, A)\right) \quad \text { for any } g=(w, c) \in G
$$

and, in particular,

$$
\widetilde{(A, a)} f(g)=f^{\prime}(g)\left(A, a+\frac{1}{2} \omega(w, A)\right) \text {. }
$$

If $h, k \in \mathfrak{g}$, then

$$
(\tilde{h} \tilde{k} f-\tilde{k} \tilde{h} f)=\widetilde{[h, k]} f
$$

The one parameter group in $G$, $e^{\text {th }}$, determined by $h=(A, a) \in \mathfrak{g}$, is given by $e^{t h}=t h=t(A, a)$.

\section{Brownian motion and heat kernel measures}

This section will closely follow [4, Section 4] except for the introduction of a certain factor of $1 / 2$ into the formalism which will simplify later formulas. Let $\{b(t)=$ $\left.\left(B(t), B_{0}(t)\right)\right\}_{t} \geqslant 0$ be a Brownian motion on $\mathfrak{g}=W \times \mathbf{C}$ with the variance determined by

$$
\mathbb{E}\left[\operatorname{Re}\langle b(s), h\rangle_{\mathfrak{g}_{C M}} \cdot \operatorname{Re}\langle b(t), k\rangle_{\mathfrak{g}_{C M}}\right]=\frac{1}{2} \operatorname{Re}\langle h, k\rangle_{\mathfrak{g}_{C M}} s \wedge t
$$


for all $s, t \in[0, \infty), h=(A, a)$, and $k:=(C, c)$, where $A, C \in H_{*}$ and $a, c \in \mathbf{C}$. (Recall the definition of $H_{*}$ from Eq. (2.15).)

Definition 4.1 The associated Brownian motion on $G$ starting at $\mathbf{e}=(0,0) \in G$ is defined to be the process

$$
g(t)=\left(B(t), B_{0}(t)+\frac{1}{2} \int_{0}^{t} \omega(B(\tau), d B(\tau))\right) .
$$

More generally, if $h \in G$, we let $g_{h}(t):=h \cdot g(t)$, the Brownian motion on $G$ starting at $h$.

Definition 4.2 Let $\mathcal{B}_{G}$ be the Borel $\sigma$-algebra on $G$ and for any $T>0$, let $\nu_{T}: \mathcal{B}_{G} \rightarrow[0,1]$ be the distribution of $g(T)$. We will call $\nu_{T}$ the heat kernel measure on $G$.

To be more explicit, the measure $v_{T}$ is the unique measure on $\left(G, \mathcal{B}_{G}\right)$ such that

$$
v_{T}(f):=\int_{G} f d \nu_{T}=\mathbb{E}[f(g(T))]
$$

for all bounded measurable functions $f: G \rightarrow \mathbb{C}$. Our next goal is to describe the generator of the process $\left\{g_{h}(t)\right\}_{t} \geqslant 0$.

Definition 4.3 A function $f: G \rightarrow \mathbb{C}$ is said to be a cylinder function if it may be written as $f=F \circ \pi_{P}$ for some $P \in \operatorname{Proj}(W)$ and some function $F: G_{P} \rightarrow \mathbb{C}$, where $G_{P}$ is defined as in Notation 1.1. We say that $f$ is a holomorphic (smooth) cylinder function if $F: G_{P} \rightarrow \mathbb{C}$ is holomorphic (smooth). We will denote the space of holomorphic (analytic) cylinder functions by $\mathcal{A}$.

Proposition 4.4 (Generator of $g_{h}$ ) If $f: G \rightarrow \mathbb{C}$ is a smooth cylinder function, let

$$
L f:=\sum_{j=1}^{\infty}\left[{\widetilde{\left(e_{j}, 0\right)}}^{2}+{\widehat{\left(i e_{j}, 0\right)}}^{2}\right] f+\sum_{j=1}^{d}\left[{\widehat{\left(0, f_{j}\right)}}^{2}+{\widehat{\left(0, i f_{j}\right)}}^{2}\right] f,
$$

where $\left\{e_{j}\right\}_{j=1}^{\infty}$ and $\left\{f_{j}\right\}_{j=1}^{d}$ are complex orthonormal bases for $\left(H,\langle\cdot, \cdot\rangle_{H}\right)$ and $\left(\mathbf{C},\langle\cdot, \cdot\rangle_{\mathbf{C}}\right)$ respectively. Then $L f$ is well defined, i.e. the sums in Eq. (4.4) are convergent and independent of the choice of bases. Moreover, for all $h \in G, \frac{1}{4} L$ is the generator for $\left\{g_{h}(t)\right\}_{t} \geqslant 0$. More precisely,

$$
M_{t}^{f}:=f\left(g_{h}(t)\right)-\frac{1}{4} \int_{0}^{t} L f\left(g_{h}(\tau)\right) d \tau
$$

is a local martingale for any smooth cylinder function, $f: G \rightarrow \mathbb{C}$. 
Proof After bearing in mind the factor of 1/2 used in defining the Brownian motion $b(t)$ in Eq. (4.1), this proposition becomes a direct consequence of Proposition 3.29 and Theorem 4.4 of [4]. Indeed, the Brownian motions in this paper are equal in distribution to the Brownian motions used in [4] after making the time change, $t \rightarrow t / 2$. It is this time change that is responsible for the $1 / 4$ factor (rather than $1 / 2$ ) in Eq. (4.5).

\subsection{Heat kernel quasi-invariance properties}

In this subsection, we are going to recall one of the key theorems from [4]. We first need a little more notation.

Let $C_{C M}^{1}$ denote the collection of $C^{1}$-paths, $g:[0,1] \rightarrow G_{C M}$. The length of $g$ is defined as

$$
\ell_{G_{C M}}(g)=\int_{0}^{1}\left\|l_{g^{-1}(s) *} g^{\prime}(s)\right\|_{\mathfrak{g}_{C M}} d s
$$

As usual, the Riemannian distance between $x, y \in G_{C M}$ is defined as

$$
d_{G_{C M}}(x, y)=\inf \left\{\ell_{G_{C M}}(g): g \in C_{C M}^{1} \ni g(0)=x \text { and } g(1)=y\right\} .
$$

Let us also recall the definition of $k(\omega)$ from [4, Eq. 7.6];

$$
\begin{aligned}
k(\omega) & =-\frac{1}{2} \sup _{\|A\|_{H_{\mathrm{Re}}}=1}\|\omega(\cdot, A)\|_{H_{\mathrm{Re}}^{*} \otimes \mathbf{C}_{\mathrm{Re}}}^{2} \\
& =-\sup _{\|A\|_{H}=1}\|\omega(\cdot, A)\|_{H^{*} \otimes \mathbf{C}}^{2} \geqslant-\|\omega\|_{H^{*} \otimes H^{*} \otimes C}^{2}>-\infty,
\end{aligned}
$$

wherein we have used [4, Lemma 3.17] in the second equality. It is known by Fernique's or Skhorohod's theorem that $\|\omega\|_{2}^{2}=\|\omega\|_{H^{*} \otimes H^{*} \otimes C}^{2}<\infty$, see [4, Proposition 3.14] for details.

Theorem 4.5 For all $h \in G_{C M}$ and $T>0$, the measures, $v_{T} \circ l_{h}^{-1}$ and $v_{T} \circ r_{h}^{-1}$, are absolutely continuous relative to $v_{T}$. Let $Z_{h}^{l}:=\frac{d\left(\nu_{T} \circ l_{h}^{-1}\right)}{d \nu_{T}}$ and $Z_{h}^{r}:=\frac{d\left(v_{T} \circ r_{h}^{-1}\right)}{d \nu_{T}}$ be the respective Randon-Nikodym derivatives, $k(\omega)$ is given in Eq. (4.8), and

$$
c(t):=\frac{t}{e^{t}-1} \quad \text { for any } t \in \mathbb{R}
$$

with the convention that $c(0)=1$. Then for all $1 \leqslant p<\infty, Z_{h}^{l}$ and $Z_{h}^{r}$ are both in $L^{p}\left(v_{T}\right)$ and satisfy the estimate

$$
\left\|Z_{h}^{*}\right\|_{L^{p}\left(v_{T}\right)} \leqslant \exp \left(\frac{c(k(\omega) T / 2)(p-1)}{T} d_{G_{C M}}^{2}(\mathbf{e}, h)\right),
$$

where $*=l$ or $*=r$. 
Proof This is [4, Theorem 8.1] (also see [4, Corollary 7.3]) with the modification that $T$ should be replaced by $T / 2$. This is again due to the fact that the Brownian motions in this paper are equal in distribution to those in [4] after making the time change, $t \rightarrow t / 2$.

It might be enlightening to note here that we call $G_{C M}$ the Cameron-Martin subgroup not only because it is constructed from the Cameron-Martin subspace, $H$, but also because it has properties similar to $H$. In particular, the following statement holds.

Proposition 4.6 The heat kernel measure does not charge $G_{C M}$, i.e. $v_{T}\left(G_{C M}\right)=0$.

Proof Note that for a bounded measurable function $f: W \times C \rightarrow \mathbb{C}$ that depends only on the the first component in $W \times C$, that is, $f(w, c)=f(w)$ we have

$$
\int_{G} f(w) d \nu_{T}(w, c)=\mathbb{E}[f(B(T))]=\int_{W} f(w) d \mu_{T}(w) .
$$

Note that for the projection $\pi: W \times C \rightarrow W, \pi(w, c)=w$ we have $\pi_{*} \nu_{T}=\mu_{T}$ and therefore

$$
v_{T}\left(G_{C M}\right)=v_{T}\left(\pi^{-1}(H)\right)=\pi_{*} v_{T}(H)=\mu_{T}(H)=0 .
$$

For later purposes, we would like to introduce the heat operator, $S_{T}:=e^{T L / 4}$, acting on $L^{p}\left(G, v_{T}\right)$. To motivate our definition, suppose $f: G \rightarrow \mathbb{C}$ is a smooth cylinder function and suppose we can make sense of $u(t, y)=\left(e^{(T-t) L / 4} f\right)(y)$. Then working formally, by Itô's formula, Eq. (4.5), and the left invariance of $L$, we expect $u(t, h g(t))$ to be a martingale for $0 \leqslant t \leqslant T$ and in particular,

$$
\mathbb{E}[f(h g(T))]=\mathbb{E}[u(T, h g(T))]=\mathbb{E}[u(0, h g(0))]=\left(e^{T L / 4} f\right)(h) .
$$

Definition 4.7 For $T>0, p \in(1, \infty]$, and $f \in L^{p}\left(G, v_{T}\right)$, let $S_{T} f: G_{C M} \rightarrow \mathbb{C}$ be defined by

$$
\left(S_{T} f\right)(h)=\int_{G} f(h \cdot g) d \nu_{T}(g)=\mathbb{E}[f(h g(T))] .
$$

The following result is a simple corollary of Theorem 4.5 and Hölder's inequality along with the observation that $p^{\prime}-1=(p-1)^{-1}$, where $p^{\prime}$ is the conjugate exponent to $p \in(1, \infty]$.

Corollary 4.8 If $p>1, T>0, f \in L^{p}\left(G, v_{T}\right), h \in G_{C M}$, and

$$
Z_{h}^{l} \in L^{\infty-}\left(v_{T}\right):=\cap_{1 \leqslant q<\infty} L^{q}\left(v_{T}\right)
$$


is as in Theorem 4.5, then $S_{T} f$ is well defined and may be computed as

$$
\left(S_{T} f\right)(h)=\int_{G} f(g) Z_{h}^{l}(g) d v_{T}(g)
$$

Moreover, we have the following pointwise "Gaussian" bounds

$$
\left|\left(S_{T} f\right)(h)\right| \leqslant\|f\|_{L^{p}\left(v_{T}\right)} \exp \left(\frac{c(k(\omega) T / 2)}{T(p-1)} d_{G_{C M}}^{2}(\mathbf{e}, h)\right) .
$$

We will see later that when $f$ is "holomorphic" and $p=2$, the above estimate in Eq. (4.14) may be improved to

$$
\left|\left(S_{T} f\right)(h)\right| \leqslant\|f\|_{L^{2}\left(v_{T}\right)} \exp \left(\frac{1}{2 T} d_{G_{C M}}^{2}(\mathbf{e}, h)\right) \text { for any } h \in G_{C M} .
$$

This bound is a variant of Bargmann's pointwise bounds (see [1, Eq. (1.7)] and [6, Eq. (5.4)]).

Lemma 4.9 Let $T>0$ and suppose that $f: G \rightarrow \mathbb{C}$ is a continuous and in $L^{p}\left(v_{T}\right)$ for some $p>1$. Then $S_{T} f: G_{C M} \rightarrow \mathbb{C}$ is continuous.

Proof For $q \in(1, p)$ and $h \in G_{C M}$ we have by Hölder's inequality and Theorem 4.5 that

$$
\begin{aligned}
\mathbb{E}|f(h g(T))|^{q} & =v_{T}\left(|f|^{q} Z_{h}^{l}\right) \leqslant\|f\|_{L^{p}\left(v_{T}\right)}^{q / p} \cdot\left\|Z_{h_{n}}^{l}\right\|_{L^{\frac{p}{p-q}}\left(\nu_{T}\right)} \\
& \leqslant\|f\|_{L^{p}\left(\nu_{T}\right)}^{q / p} \exp \left(\frac{c(k(\omega) T / 2) q}{T(p-q)} d_{G_{C M}}^{2}(\mathbf{e}, h)\right)
\end{aligned}
$$

Hence if $\left\{h_{n}\right\}_{n=1}^{\infty} \subset G_{C M}$ is a sequence converging to $h \in G_{C M}$, it follows that

$$
\sup _{n} \mathbb{E}\left|f\left(h_{n} g(T)\right)\right|^{q} \leqslant\|f\|_{L^{p}\left(v_{T}\right)}^{q / p} \exp \left(\frac{c(k(\omega) T / 2) q}{T(p-q)} \sup _{n} d_{G_{C M}}^{2}\left(\mathbf{e}, h_{n}\right)\right)<\infty
$$

which implies that $\left\{f\left(h_{n} g(T)\right)\right\}_{n=1}^{\infty}$ is uniformly integrable. Since by continuity of $f$, $\lim _{n \rightarrow \infty} f\left(h_{n} g(T)\right)=f(h g(T))$, we may pass to the limit under the expectation to find

$$
\lim _{n \rightarrow \infty} S_{T} f\left(h_{n}\right)=\lim _{n \rightarrow \infty} \mathbb{E} f\left(h_{n} g(T)\right)=\mathbb{E}[f(h g(T))]=S_{T} f(h)
$$


4.2 Finite dimensional approximations

Notation 4.10 For each $P \in \operatorname{Proj}(W)$, let $g_{P}(t)$ denote the $G_{P}$-valued Brownian motion defined by

$$
g_{P}(t)=\left(P B(t), B_{0}(t)+\frac{1}{2} \int_{0}^{t} \omega(P B(\tau), d P B(\tau))\right) .
$$

Also, for any $t>0$, let $v_{t}^{P}:=\operatorname{Law}\left(g_{P}(t)\right)$ be the corresponding heat kernel measure on $G_{P}$.

The following Theorem is a restatement of [4, Theorem 4.16].

Theorem 4.11 (Integrated heat kernel bounds) Suppose that $\rho^{2}: G \rightarrow[0, \infty)$ be defined as

$$
\rho^{2}(w, c):=\|w\|_{W}^{2}+\|c\|_{\mathbf{C}}
$$

Then there exists a $\delta>0$ such that for all $\varepsilon \in(0, \delta)$ and $T>0$

$$
\sup _{P \in \operatorname{Proj}(W)} \mathbb{E}\left[e^{\frac{\varepsilon}{T} \rho^{2}\left(g_{P}(T)\right)}\right]<\infty \quad \text { and } \quad \int_{G} e^{\frac{\varepsilon}{T} \rho^{2}(g)} d \nu_{T}(g)<\infty .
$$

Proposition 4.12 Let $P_{n} \in \operatorname{Proj}(W)$ such that $\left.P_{n}\right|_{H} \uparrow I_{H}$ on $H$ and let $g_{n}(T):=$ $g_{P_{n}}(T)$. Further suppose that $\delta>0$ is as in Theorem 4.11, $p \in[1, \infty)$, and $f: G \rightarrow \mathbb{C}$ is a continuous function such that

$$
|f(g)| \leqslant C e^{\varepsilon \rho^{2}(g) /(p T)} \quad \text { for all } g \in G
$$

for some $\varepsilon \in(0, \delta)$. Then $f \in L^{p}\left(v_{T}\right)$ and for all $h \in G$ we have

$$
\lim _{n \rightarrow \infty} \mathbb{E}\left|f(h g(T))-f\left(h g_{n}(T)\right)\right|^{p}=0,
$$

and

$$
\lim _{n \rightarrow \infty} \mathbb{E}\left|f(g(T) h)-f\left(g_{n}(T) h\right)\right|^{p}=0
$$

Proof If $q \in(p, \infty)$ is sufficiently close to $p$ so that $q p^{-1} \varepsilon<\delta$, then

$$
\sup _{n} \mathbb{E}\left|f\left(g_{n}(T)\right)\right|^{q} \leqslant C^{q} \sup _{n} \mathbb{E}\left[e^{p^{-1} q \varepsilon \rho^{2}(g) / T}\right]
$$

which is finite by Theorem 4.11. This shows that $\left\{\left|f\left(g_{n}(T)\right)\right|^{p}\right\}_{n=1}^{\infty}$ is uniformly integrable. As a consequence of [4, Lemma 4.7] and the continuity of $f$, we also know 
that $f\left(g_{n}(T)\right) \rightarrow f(g(T))$ in probability as $n \rightarrow \infty$. Thus we have shown Eqs. (4.22) and (4.23) hold when $h=\mathbf{e}=0$. Now suppose that $g=(w, c)$ and $h=(A, a)$ are in $G$. Then for all $\alpha>0$,

$$
\begin{aligned}
\rho^{2}(g h) & =\|w+A\|_{W}^{2}+\left\|a+c+\frac{1}{2} \omega(w, A)\right\|_{\mathbf{C}} \\
& \leqslant\|w\|_{W}^{2}+\|A\|_{W}^{2}+2\|A\|_{W}\|w\|_{W}+\|a\|_{\mathbf{C}}+\|c\|_{\mathbf{C}}+\frac{1}{2}\|\omega(w, A)\|_{\mathbf{C}} \\
& \leqslant \rho^{2}(g)+\rho^{2}(h)+C\|A\|_{W}\|w\|_{W} \\
& \leqslant \rho^{2}(g)+\rho^{2}(h)+\frac{C}{2}\left[\alpha^{-1}\|A\|_{W}^{2}+\alpha\|w\|_{W}^{2}\right] \\
& \leqslant\left(1+\frac{C \alpha}{2}\right) \rho^{2}(g)+\left(1+\frac{C}{2 \alpha}\right) \rho^{2}(h)
\end{aligned}
$$

where $C:=\left(2+\frac{1}{2}\|\omega\|_{0}\right)$. As Eq. (4.24) is invariant under interchanging $g$ and $h$ the same bound also hold for $\rho^{2}(h g)$. By choosing $\alpha>0$ sufficiently small so that $\left(1+\frac{C \alpha}{2}\right) \varepsilon<\delta$, we see that $g \rightarrow f(g h)$ and $g \rightarrow f(h g)$ satisfy the same type of bound as in Eq. (4.21) for $g \rightarrow f(g)$. Therefore, by the first paragraph, we have now verified Eqs. (4.22) and (4.23) hold for any $h \in G$.

\section{Holomorphic functions on $G$ and $G_{C M}$}

We will begin with a short summary of the results about holomorphic functions on Banach spaces that will be needed in this paper.

5.1 Holomorphic functions on Banach spaces

Let $X$ and $Y$ be two complex Banach space and for $a \in X$ and $\delta>0$ let

$$
B_{X}(a, \delta):=\left\{x \in X:\|x-a\|_{X}<\delta\right\}
$$

be the open ball in $X$ with center $a$ and radius $\delta$.

Definition 5.1 (Hille and Phillips [17, Definition 3.17.2, p. 112.]) Let $\mathcal{D}$ be an open subset of $X$. A function $u: \mathcal{D} \rightarrow Y$ is said to be holomorphic (or analytic) if the following two conditions hold.

(1) $u$ is locally bounded, namely for all $a \in \mathcal{D}$ there exists an $r_{a}>0$ such that

$$
M_{a}:=\sup \left\{\|u(x)\|_{Y}: x \in B_{X}\left(a, r_{a}\right)\right\}<\infty .
$$

(2) The function $u$ is complex Gâteaux differentiable on $\mathcal{D}$, i.e. for each $a \in \mathcal{D}$ and $h \in X$, the function $\lambda \rightarrow u(a+\lambda h)$ is complex differentiable at $\lambda=0 \in \mathbb{C}$.

(Holomorphic and analytic will be considered to be synonymous terms for the purposes of this paper.) 
The next theorem gathers together a number of basic properties of holomorphic functions which may be found in [17]. (Also see [15].) One of the key ingredients to all of these results is Hartog's theorem, see [17, Theorem 3.15.1].

Theorem 5.2 If $u: \mathcal{D} \rightarrow Y$ is holomorphic, then there exists a function $u^{\prime}: \mathcal{D} \rightarrow$ $\operatorname{Hom}(X, Y)$, the space of bounded complex linear operators from $X$ to $Y$, satisfying

(1) If $a \in \mathcal{D}, x \in B_{X}\left(a, r_{a} / 2\right)$, and $h \in B_{X}\left(0, r_{a} / 2\right)$, then

$$
\left\|u(x+h)-u(x)-u^{\prime}(x) h\right\|_{Y} \leqslant \frac{4 M_{a}}{r_{a}\left(r_{a}-2\|h\|_{X}\right)}\|h\|_{X}^{2} .
$$

In particular, $u$ is continuous and Frechét differentiable on $\mathcal{D}$.

(2) The function $u^{\prime}: \mathcal{D} \rightarrow \operatorname{Hom}(X, Y)$ is holomorphic.

Remark 5.3 By applying Theorem 5.2 repeatedly, it follows that any holomorphic function, $u: \mathcal{D} \rightarrow Y$ is Frechét differentiable to all orders and each of the Frechét differentials are again holomorphic functions on $\mathcal{D}$.

Proof By [17, Theorem 26.3.2 on p. 766.], for each $a \in \mathcal{D}$ there is a linear operator, $u^{\prime}(a): X \rightarrow Y$ such that $d u(a+\lambda h) /\left.d \lambda\right|_{\lambda=0}=u^{\prime}(a) h$. The Cauchy estimate in Theorem 3.16 .3 (with $n=1$ ) of [17] implies that if $a \in \mathcal{D}, x \in B_{X}\left(a, r_{a} / 2\right)$ and $h \in B_{X}\left(0, r_{a} / 2\right)$ (so that $x+h \in B_{X}\left(a, r_{a}\right)$ ), then $\left\|u^{\prime}(x) h\right\|_{Y} \leqslant M_{a}$. It follows from this estimate that

$$
\sup \left\{\left\|u^{\prime}(x)\right\|_{\operatorname{Hom}(X, Y)}: x \in B_{X}\left(a, r_{a} / 2\right)\right\} \leqslant 2 M_{a} / r_{a} .
$$

and hence that $u^{\prime}: \mathcal{D} \rightarrow \operatorname{Hom}(X, Y)$ is a locally bounded function. The estimate in Eq. (5.1) appears in the proof of the Theorem 3.17.1 in [17] which completes the proof of item 1 .

To prove item 2. we must show $u^{\prime}$ is Gâteaux differentiable on $\mathcal{D}$. We will in fact show more, namely, that $u^{\prime}$ is Frechét differentiable on $\mathcal{D}$. Given $h \in X$, let $F_{h}: \mathcal{D} \rightarrow Y$ be defined by $F_{h}(x):=u^{\prime}(x) h$. According to [17, Theorem 26.3.6], $F_{h}$ is holomorphic on $\mathcal{D}$ as well. Moreover, if $a \in \mathcal{D}$ and $x \in B\left(a, r_{a} / 2\right)$ we have by Eq. (5.2) that

$$
\left\|F_{h}(x)\right\|_{Y} \leqslant 2 M_{a}\|h\|_{X} / r_{a}
$$

So applying the estimate in Eq. (5.1) to $F_{h}$, we learn that

$$
\left\|F_{h}(x+k)-F_{h}(x)-F_{h}^{\prime}(x) k\right\|_{Y} \leqslant \frac{4\left(2 M_{a}\|h\|_{X} / r_{a}\right)}{\frac{r_{a}}{2}\left(\frac{r_{a}}{2}-2\|k\|_{X}\right)} \cdot\|k\|_{X}^{2}
$$

for $x \in B\left(a, r_{a} / 4\right)$ and $\|k\|_{X}<r_{a} / 4$, where

$$
F_{h}^{\prime}(x) k=\left.\frac{d}{d \lambda}\right|_{0} F_{h}(x+\lambda k)=\left.\frac{d}{d \lambda}\right|_{0} u^{\prime}(x+\lambda k) h=:\left(\delta^{2} u\right)(x ; h, k) .
$$


Again by [17, Theorem 26.3.6], for each fixed $x \in \mathcal{D},\left(\delta^{2} u\right)(x ; h, k)$ is a continuous symmetric bilinear form in $(h, k) \in X \times X$. Taking the supremum of Eq. (5.3) over those $h \in X$ with $\|h\|_{X}=1$, we may conclude that

$$
\begin{aligned}
& \left\|u^{\prime}(x+k)-u^{\prime}(x)-\delta^{2} u(x ; \cdot, k)\right\|_{\operatorname{Hom}(X, Y)} \\
& =\sup _{\|h\|_{X}=1}\left\|F_{h}(x+k)-F_{h}(x)-F_{h}^{\prime}(x) k\right\|_{Y} \\
& \leqslant \frac{4\left(2 M_{a} / r_{a}\right)}{\frac{r_{a}}{2}\left(\frac{r_{a}}{2}-2\|k\|_{X}\right)}\|k\|_{X}^{2} .
\end{aligned}
$$

This estimate shows $u^{\prime}$ is Frechét differentiable with $u^{\prime \prime}(x) \in \operatorname{Hom}(X, \operatorname{Hom}(X, Y))$ being given by $u^{\prime \prime}(x) k=\left(\delta^{2} u\right)(x ; \cdot, k) \in \operatorname{Hom}(X, Y)$ for all $k \in X$ and $x \in \mathcal{D}$.

\subsection{Holomorphic functions on $G$ and $G_{C M}$}

For the purposes of this section, let $G_{0}=G$ and $\mathfrak{g}_{0}=\mathfrak{g}$ or $G_{0}=G_{C M}$ and $\mathfrak{g}_{0}=\mathfrak{g}_{C M}$. Also for $g, h \in \mathfrak{g}$, let (as usual) $a d_{g} h:=[g, h]$.

Lemma 5.4 For each $g \in G_{0}, l_{g}: G_{0} \rightarrow G_{0}$ is holomorphic in the $\|\cdot\|_{\mathfrak{g}_{0} \text {-topol- }}$ ogy. Moreover, a function $u: G_{0} \rightarrow \mathbb{C}$ defined in a neighborhood of $g \in G_{0}$ is Gâteaux (Frechét) differentiable at $g$ iff $u \circ l_{g}$ is Gâteaux (Frechét) differentiable at 0. In addition, if $u$ is Frechét differentiable at $g$, then

$$
\left(u \circ l_{g}\right)^{\prime}(0) h=u^{\prime}(g)\left(h+\frac{1}{2}[g, h]\right) .
$$

(See [13, Theorem 5.7] for an analogous result in the context of path groups.)

Proof Since

$$
l_{g}(h)=g h=g+h+\frac{1}{2}[g, h]=g+\left(I d_{\mathfrak{g}_{0}}+\frac{1}{2} a d_{g}\right) h,
$$

it is easy to see that $l_{g}$ is holomorphic and $l_{g}^{\prime}$ is the constant function equal to $I d_{\mathfrak{g}_{0}}+$ $\frac{1}{2} a d_{g} \in \operatorname{End}\left(\mathfrak{g}_{0}\right)$. Using $a d_{g}^{2}=0$ or the fact that $l_{g}^{-1}=l_{g^{-1}}$, we see that $l_{g}^{\prime}$ is invertible and that

$$
l_{g}^{\prime-1}=\left(I d_{\mathfrak{g}_{0}}+\frac{1}{2} a d_{g}\right)^{-1}=I d_{\mathfrak{g}_{0}}-\frac{1}{2} a d_{g} .
$$

These observations along with the chain rule imply the Frechét differentiability statements of the lemma and the identity in Eq. (5.4).

If $u$ is Gâteaux differentiable at $g, h \in \mathfrak{g}_{0}$, and $k:=h+\frac{1}{2}[g, h]$, then

$$
\left.\frac{d}{d \lambda}\right|_{0} u \circ l_{g}(\lambda h)=\left.\frac{d}{d \lambda}\right|_{0} u(g \cdot(\lambda h))=\left.\frac{d}{d \lambda}\right|_{0} u(g+\lambda k)
$$


and the existence of $\left.\frac{d}{d \lambda}\right|_{0} u(g+\lambda k)$ implies the existence of $\left.\frac{d}{d \lambda}\right|_{0} u \circ l_{g}(\lambda h)$. Conversely, if $u \circ l_{g}$ is Gâteaux differentiable at $0, h \in \mathfrak{g}_{0}$, and

$$
k:=h-\frac{1}{2}[g, h]=\left(I d_{\mathfrak{g}_{0}}+\frac{1}{2} a d_{g}\right)^{-1} h,
$$

then

$$
l_{g}(\lambda k)=g+\lambda\left(I d_{\mathfrak{g}_{0}}+\frac{1}{2} a d_{g}\right) k=g+\lambda h .
$$

So the existence of $\left.\frac{d}{d \lambda}\right|_{0}\left(u \circ l_{g}\right)(\lambda k)$ implies the existence of $\left.\frac{d}{d \lambda}\right|_{0} u(g+\lambda h)$.

Corollary 5.5 A function $u: G_{0} \rightarrow \mathbb{C}$ is holomorphic iff it is locally bounded and $h \rightarrow u\left(g e^{h}\right)=u(g \cdot h)$ is Gâteaux (Frechét) differentiable at 0 for all $g \in G_{0}$. Moreover, if $u$ is holomorphic and $h \in \mathfrak{g}_{0}$, then

$$
(\tilde{h} u)(g)=\left.\frac{d}{d \lambda}\right|_{0} u\left(g e^{\lambda h}\right)=u^{\prime}(g)(h+[g, h])
$$

is holomorphic as well.

Notation 5.6 The space of globally defined holomorphic functions on $G$ and $G_{C M}$ will be denoted by $\mathcal{H}(G)$ and $\mathcal{H}\left(G_{C M}\right)$ respectively.

Notice that the space $\mathcal{A}$ of holomorphic cylinder functions as described in Definition 4.3 is contained in $\mathcal{H}(G)$. Also observe that a simple induction argument using Corollary 5.5 allows us to conclude that $\tilde{h}_{1} \ldots \tilde{h}_{n} u \in \mathcal{H}\left(G_{0}\right)$ for all $u \in \mathcal{H}\left(G_{0}\right)$ and $h_{1}, \ldots, h_{n} \in \mathfrak{g}_{0}$.

Proposition 5.7 If $f \in \mathcal{H}(G)$ and $h \in \mathfrak{g}$, then $\widetilde{i h} f=i \tilde{h} f, \tilde{i h} \bar{f}=-i \tilde{h} \bar{f}$,

$$
\begin{gathered}
{\left[(\tilde{i h})^{2}+\tilde{h}^{2}\right] f=0, \text { and }} \\
\left(\tilde{h}^{2}+\tilde{i h}^{2}\right)|f|^{2}=4|\tilde{h} f|^{2} .
\end{gathered}
$$

Proof The first assertions are directly related to the definition of $f$ being holomorphic. Using the identity $\widetilde{i h} f=i \tilde{h} f$ twice implies Eq. (5.5). Equation (5.6) is a consequence of summing the following two identities

$$
\tilde{h}^{2}|f|^{2}=\tilde{h}(f \cdot \bar{f})=\tilde{h}^{2} f \cdot \bar{f}+f \cdot \tilde{h}^{2} \bar{f}+2 \tilde{h} f \cdot \tilde{h} \bar{f}
$$

and

$$
\begin{aligned}
\widetilde{i h}^{2}|f|^{2} & =\widetilde{i h}(f \cdot \bar{f})=\widetilde{i h}^{2} f \cdot \bar{f}+f \cdot \widetilde{i h}^{2} \bar{f}+2 \widetilde{i h} f \cdot \tilde{i h} \bar{f} \\
& =-\widetilde{h}^{2} f \cdot \bar{f}-f \cdot \widetilde{h}^{2} \bar{f}+2 \widetilde{h} f \cdot \widetilde{h} \bar{f}
\end{aligned}
$$

and using $\widetilde{h} \bar{f}=\overline{\widetilde{h} f}$. 
Corollary 5.8 Let $L$ be as in Proposition 4.4. Suppose that $f: G \rightarrow \mathbb{C}$ is a holomorphic cylinder function (i.e. $f \in \mathcal{A}$ ), then $L f=0$ and

$$
L|f|^{2}=\sum_{h \in \Gamma}|\tilde{h} f|^{2}
$$

where $\Gamma$ is an orthonormal basis for $\mathfrak{g}_{C M}$ of the form

$$
\Gamma=\Gamma_{e} \cup \Gamma_{f}=\left\{\left(e_{j}, 0\right)\right\}_{j=1}^{\infty} \cup\left\{\left(0, f_{j}\right)\right\}_{j=1}^{d}
$$

with $\left\{e_{j}\right\}_{j=1}^{\infty}$ and $\left\{f_{j}\right\}_{j=1}^{d}$ being complex orthonormal bases for $H$ and $\mathbf{C}$ respectively. Proof These assertions follow directly form Eqs. (4.4), (5.5), and (5.6).

Formally, if $f: G \rightarrow \mathbb{C}$ is a holomorphic function, then $e^{T L / 4} f=f$ and therefore we should expect $S_{T} f=\left.f\right|_{G_{C M}}$ where $S_{T}$ is defined in Definition 4.7. Theorem 5.9 below is a precise version of this heuristic.

Theorem 5.9 Suppose $p \in(1, \infty)$ and $f: G \rightarrow \mathbb{C}$ is a continuous function such that $\left.f\right|_{G_{C M}} \in \mathcal{H}\left(G_{C M}\right)$ and there exists $P_{n} \in \operatorname{Proj}(W)$ such that $\left.P_{n}\right|_{H} \uparrow I_{H}$, then

$$
\|f\|_{L^{p}\left(v_{T}\right)} \leqslant \sup _{n}\|f\|_{L^{p}}\left(G_{P_{n}}, v_{T}^{P_{n}}\right) .
$$

If we further assume that

$$
\sup _{n}\|f\|_{L^{p}}\left(G_{P_{n}}, v_{T}^{P_{n}}\right)<\infty
$$

then $f \in L^{p}\left(v_{T}\right), S_{T} f=\left.f\right|_{G_{C M}}$, and $f$ satisfies the Gaussian bounds

$$
|f(h)| \leqslant\|f\|_{L^{p}\left(\nu_{T}\right)} \exp \left(\frac{c(k(\omega) T / 2)}{T(p-1)} d_{G_{C M}}^{2}(\mathbf{e}, h)\right) \text { for any } h \in G_{C M} .
$$

Proof According to [4, Lemma 4.7], by passing to a subsequence if necessary, we may assume that $g_{P_{n}}(T) \rightarrow g(T)$ almost surely. Hence an application of Fatou's lemma implies Eq. (5.9). In particular, if we assume Eq. (5.10) holds, then $f \in L^{p}\left(v_{T}\right)$ and so $S_{T} f$ is well defined.

Now suppose that $P \in \operatorname{Proj}(W)$ and $h \in G_{P}$. Working exactly as in the proof of Lemma 4.9 , we find for any $q \in(1, p)$ that

$$
\mathbb{E}\left|f\left(h g_{P}(T)\right)\right|^{q} \leqslant\|f\|_{L^{p}\left(G_{P}, v_{T}^{P}\right)}^{q / p} \exp \left(\frac{c\left(k_{P}(\omega) T / 2\right) q}{T(q-p)} d_{G_{P}}^{2}(\mathbf{e}, h)\right),
$$

where $d_{G_{P}}(\cdot, \cdot)$ is the Riemannian distance on $G_{P}$ and (see [4, Eq. (5.13)]),

$$
k_{P}(\omega):=-\frac{1}{2} \sup \left\{\|\omega(\cdot, A)\|_{(P H)^{*} \otimes \mathbf{C}}^{2}: A \in P H,\|A\|_{P H}=1\right\} .
$$


Observe that $k_{P}(\omega) \geqslant k(\omega)$ and therefore, as $c$ is a decreasing function, $c(k(\omega)) \geqslant$ $c\left(k_{P}(\omega)\right)$. Let $m \in \mathbb{N}$ be given and $h \in G_{P_{m}}$. Then for $n \geqslant m$ we have from Eq. (5.12) that

$$
\begin{aligned}
\mathbb{E}\left|f\left(h g_{P_{n}}(T)\right)\right|^{q} & \leqslant\|f\|_{L^{p}\left(G_{P_{n}}, v_{T}^{P_{n}}\right)}^{q / p} \exp \left(\frac{c\left(k_{P_{n}}(\omega) T / 2\right) q}{T(q-p)} d_{G_{P_{n}}}^{2}(\mathbf{e}, h)\right) \\
& \leqslant\|f\|_{L^{p}\left(G_{P_{n}}, v_{T}^{P_{n}}\right)}^{q / p} \exp \left(\frac{c(k(\omega) T / 2) q}{T(q-p)} d_{G_{P_{m}}}^{2}(\mathbf{e}, h)\right)
\end{aligned}
$$

wherein in the last inequality we have used $c(k(\omega)) \geqslant c\left(k_{P}(\omega)\right)$ and the fact that $d_{G_{P_{n}}}^{2}(\mathbf{e}, h)$ is decreasing in $n \geqslant m$. Hence it follows that $\sup _{n \geqslant m} \mathbb{E}\left|f\left(h_{P_{n}}(T)\right)\right|^{q}<\infty$ and thus that $\left\{f\left(h g_{P_{n}}(T)\right)\right\}_{n} \geqslant m$ is uniformly integrable. Therefore,

$$
S_{T} f(h)=\mathbb{E} f(h g(T))=\lim _{n \rightarrow \infty} \mathbb{E} f\left(h g_{P_{n}}(T)\right)=\lim _{n \rightarrow \infty} \int_{G_{P_{n}}} f(h x) d \nu_{T}^{P_{n}}(x) .
$$

On the other hand by [4, Lemma 4.8] (with $T$ replaced by $T / 2$ because of our normalization in Eq. (4.1)), $v_{T}^{P_{n}}$ is the heat kernel measure on $G_{P_{n}}$ based at $\mathbf{e} \in G_{P_{n}}$, i.e. $v_{T}^{P_{n}}(d x)=p_{T / 2}^{P_{n}}(e, x) d x$, where $d x$ is the Riemannian volume measure (equal to a Haar measure) on $G_{P_{n}}$ and $p_{T}^{P_{n}}(x, y)$ is the heat kernel on $G_{P_{n}}$. Since $\left.f\right|_{G_{P_{n}}}$ is holomorphic, the previous observations allow us to apply [5, Proposition 1.8] to conclude that

$$
\int_{G_{P_{n}}} f(h x) d v_{T}^{P_{n}}(x)=f(h) \text { for all } n \geqslant m .
$$

As $m \in \mathbb{N}$ was arbitrary, combining Eqs. (5.14) and (5.15) implies that $S_{T} f(h)=f(h)$ for all $h \in G_{0}:=\cup_{m \in \mathbb{N}} G_{P_{m}}$. Recall from Lemma 4.9 that $S_{T} f: G_{C M} \rightarrow \mathbb{C}$ is continuous and from the proof of [4, Theorem 8.1] that $G_{0}$ is a dense subgroup of $G_{C M}$. Therefore we may conclude that in fact $S_{T} f(h)=f(h)$ for all $h \in G_{C M}$. The Gaussian bound now follows immediately from Corollary 4.8 .

Corollary 5.10 Suppose that $\delta>0$ is as in Theorem 4.11 and $f: G \rightarrow \mathbb{C}$ is a continuous function such that $\left.f\right|_{G_{C M}}$ is holomorphic and $|f| \leqslant C e^{\varepsilon \rho^{2} /(p T)}$ for some $\varepsilon \in[0, \delta)$. Then $f \in L^{p}\left(v_{T}\right), S_{T} f=f$, and the Gaussian bounds in Eq. (5.11) hold.

Proof By Theorem 4.11, the given function $f$ verifies Eq. (5.10) for any choice of $\left\{P_{n}\right\}_{n=1}^{\infty} \subset \operatorname{Proj}(W)$ with $\left.P_{n}\right|_{H} \uparrow P$ strongly as $n \uparrow \infty$. Hence Theorem 5.9 is applicable.

As a simple consequence of Corollary 5.10, we know that $\mathcal{P} \subset L^{p}\left(v_{T}\right)$ (see Definition 1.6) and that $\left(S_{T} p\right)(h)=p(h)$ for all $h \in G_{C M}$ and $p \in \mathcal{P}$.

Notation 5.11 For $T>0$ and $1 \leqslant p<\infty$, let $\mathcal{A}_{T}^{p}$ and $\mathcal{H}_{T}^{p}(G)$ denote the $L^{p}\left(v_{T}\right)-$ closure of $\mathcal{A} \cap L^{p}\left(v_{T}\right)$ and $\mathcal{P}$, where $\mathcal{A}$ and $\mathcal{P}$ denote the holomorphic cylinder 
functions (see Definition 4.3) and holomorphic cylinder polynomials on $G$ respectively.

Theorem 5.12 For all $T>0$ and $p \in(1, \infty)$, we have $S_{T}\left(\mathcal{H}_{T}^{p}(G)\right) \subset \mathcal{H}\left(G_{C M}\right)$.

Proof Let $f \in \mathcal{H}_{T}^{p}(G)$ and $p_{n} \in \mathcal{P}$ such that $\lim _{n \rightarrow \infty}\left\|f-p_{n}\right\|_{L^{p}\left(\nu_{T}\right)}=0$. If $h \in G_{C M}$, then by Corollary 4.8

$$
\begin{aligned}
\left|S_{T} f(h)-p_{n}(h)\right| & =\left|S_{T}\left(f-p_{n}\right)(h)\right| \\
& \leqslant\left\|f-p_{n}\right\|_{L^{p}\left(\nu_{T}\right)} \exp \left(\frac{c(k(\omega) T / 2)}{T(p-1)} d_{G_{C M}}^{2}(\mathbf{e}, h)\right) .
\end{aligned}
$$

This shows that $S_{T} f$ is the limit of $\left.p_{n}\right|_{G_{C M}} \in \mathcal{H}\left(G_{C M}\right)$ with the limit being uniform over any bounded subset of $h$ 's contained in $G_{C M}$. This is sufficient to show that $S_{T} f \in \mathcal{H}\left(G_{C M}\right)$ via an application of [17, Theorem 3.18.1].

Remark 5.13 It seems reasonable to conjecture that $\mathcal{A}_{T}^{2}=\mathcal{H}_{T}^{2}(G)$, nevertheless we do not know if these two spaces are equal! We also do not know if $S_{T} f=f$ for every $f \in \mathcal{A} \cap L^{2}\left(v_{T}\right)$. However, Theorem 5.9 does show that $S_{T} f=f$ for all $f \in \mathcal{A} \cap_{P \in \operatorname{Proj}(W)} L^{p}\left(v_{T}^{P}\right)$ with $L^{p}\left(v_{T}^{P}\right)$-norms of $f$ being bounded.

\section{The Taylor isomorphism theorem}

The main purpose of this section is to prove the Taylor isomorphism Theorem 1.5 (or Theorem 6.10). We begin with the formal development of the algebraic setup. In what follows below for a vector space $V$ we will denote the algebraic dual to $V$ by $V^{\prime}$. If $V$ happens to be a normed space, we will let $V^{*}$ denote the topological dual of $V$.

\subsection{A non-commutative Fock space}

Notation 6.1 For $n \in \mathbb{N}$ let $\mathfrak{g}_{C M}^{\otimes n}$ denote the $n$-fold algebraic tensor product of $\mathfrak{g}_{C M}$ with itself, and by convention let $\mathfrak{g}_{C M}^{\otimes 0}:=\mathbb{C}$. Also let

$$
\mathbf{T}:=\mathbf{T}\left(\mathfrak{g}_{C M}\right)=\mathbb{C} \oplus \mathfrak{g}_{C M} \oplus \mathfrak{g}_{C M}^{\otimes 2} \oplus \mathfrak{g}_{C M}^{\otimes 3} \oplus \ldots
$$

be the algebraic tensor algebra over $\mathfrak{g}_{C M}, \mathbf{T}^{\prime}$ be its algebraic dual, and $J$ be the twosided ideal in $\mathbf{T}$ generated by the elements in Eq. (1.3). The backwards annihilator of $J$ is

$$
J^{0}=\left\{\alpha \in \mathbf{T}^{\prime}: \alpha(J)=0\right\}
$$

For any $\alpha \in \mathbf{T}^{\prime}$ and $n \in \mathbb{N} \cup\{0\}$, we let $\alpha_{n}:=\left.\alpha\right|_{\mathfrak{g}_{C M}^{\otimes n}} \in\left(\mathfrak{g}_{C M}^{\otimes n}\right)^{\prime}$.

After the next definition we will be able to give numerous examples of elements in $J^{0}$. 
Definition 6.2 (Left differentials) For $f \in \mathcal{H}\left(G_{C M}\right), n \in \mathbb{N} \cup\{0\}$, and $g \in G_{C M}$, define $\hat{f}_{n}(g):=D^{n} f(g) \in\left(\mathfrak{g}_{C M}^{\otimes n}\right)^{\prime}$ by

$$
\begin{aligned}
& \left(D^{0} f\right)(g)=f(g) \text { and } \\
& \left\langle D^{n} f(g), h_{1} \otimes \cdots \otimes h_{n}\right\rangle=\left(\tilde{h}_{1} \ldots \tilde{h}_{n} f\right)(g)
\end{aligned}
$$

for all and $h_{1}, \ldots, h_{n} \in \mathfrak{g}_{0}$, where $\tilde{h} f$ is given as in Eq. (3.8) or Eq. (3.10). We will write $D f$ for $D^{1} f$ and $\hat{f}(g)$ to be the element of $\mathbf{T}\left(\mathfrak{g}_{C M}\right)^{\prime}$ determined by

$$
\langle\hat{f}(g), \beta\rangle=\left\langle\hat{f}_{n}(g), \beta\right\rangle \text { for all } \beta \in \mathfrak{g}_{C M}^{\otimes n} \text { and } n \in \mathbb{N}_{0} .
$$

Example 6.3 As a consequence of Eq. (3.11), $\hat{f}(g) \in J^{0}$ for all $f \in \mathcal{H}\left(G_{C M}\right)$ and $g \in G_{C M}$.

In order to put norms on $J^{0}$, let us equip $\mathfrak{g}_{C M}^{\otimes n}$ with the usual inner product determined by

$$
\left\langle h_{1} \otimes \cdots \otimes h_{n}, k_{1} \otimes \cdots \otimes k_{n}\right\rangle_{\mathfrak{g}_{C M}^{\otimes n}}=\prod_{j=1}^{n}\left\langle h_{j}, k_{j}\right\rangle_{\mathfrak{g}_{C M}} \text { for any } h_{i}, k_{j} \in \mathfrak{g}_{C M}
$$

For $n=0$ we let $\langle z, w\rangle_{\mathfrak{g}_{C M}^{\otimes 0}}:=z \bar{w}$ for all $z, w \in \mathfrak{g}_{C M}^{\otimes 0}=\mathbb{C}$. The inner product $\langle\cdot, \cdot\rangle_{\mathfrak{g}_{C M}^{\otimes n}}^{\otimes n}$ induces a dual inner product on $\left(\mathfrak{g}_{C M}^{\otimes n}\right)^{*}$ which we will denote by $\langle\cdot, \cdot\rangle_{n}$. The associated norm on $\left(\mathfrak{g}_{C M}^{\otimes n}\right)^{*}$ will be denoted by $\|\cdot\|_{n}$. We extend $\|\cdot\|_{n}$ to all of $\left(\mathfrak{g}_{C M}^{\otimes n}\right)^{\prime}$ by setting $\|\beta\|_{n}=\infty$ if $\beta \in\left(\mathfrak{g}_{C M}^{\otimes n}\right)^{\prime} \backslash\left(\mathfrak{g}_{C M}^{\otimes n}\right)^{*}$. If $\Gamma$ is any orthonormal basis for $\mathfrak{g}_{C M}$, then $\|\beta\|_{n}$ may be computed using

$$
\|\beta\|_{\mathfrak{g}_{C M}^{\otimes n}}^{2}:=\sum_{h_{1}, \ldots, h_{n} \in \Gamma}\left|\left\langle\beta, h_{1} \otimes \cdots \otimes h_{n}\right\rangle\right|^{2} .
$$

Definition 6.4 (Non-commutative Fock space) Given $T>0$ and $\alpha \in J^{0}\left(\mathfrak{g}_{C M}\right)$, let

$$
\|\alpha\|_{J_{T}^{0}\left(\mathfrak{g}_{C M}\right)}^{2}:=\sum_{n=0}^{\infty} \frac{T^{n}}{n !}\left\|\alpha_{n}\right\|_{n}^{2}
$$

Further let

$$
J_{T}^{0}\left(\mathfrak{g}_{C M}\right):=\left\{\alpha \in J^{0}\left(\mathfrak{g}_{C M}\right):\|\alpha\|_{J_{T}^{0}\left(\mathfrak{g}_{C M}\right)}^{2}<\infty\right\} .
$$

The space, $J_{T}^{0}\left(\mathfrak{g}_{C M}\right)$, is then a Hilbert space when equipped with the inner product

$$
\langle\alpha, \beta\rangle_{J_{T}^{0}\left(\mathfrak{g}_{C M}\right)}=\sum_{n=0}^{\infty} \frac{T^{n}}{n !}\left\langle\alpha_{n}, \beta_{n}\right\rangle_{n} \quad \text { for any } \alpha, \beta \in J_{T}^{0}\left(\mathfrak{g}_{C M}\right) .
$$


6.2 The Taylor isomorphism

Lemma 6.5 Let $f \in \mathcal{H}\left(G_{C M}\right)$ and $T>0$ and suppose that $\left\{P_{n}\right\}_{n=1}^{\infty} \subset \operatorname{Proj}(W)$ is a sequence such that $\left.P_{n}\right|_{\mathfrak{g}_{C M}} \uparrow I_{\mathfrak{g}_{C M}}$ as $n \rightarrow \infty$. Then

$$
\lim _{n \rightarrow \infty}\|\hat{f}(\mathbf{e})\|_{J_{T}^{0}\left(\mathfrak{g}_{P_{n}}\right)}=\|\hat{f}(\mathbf{e})\|_{J_{T}^{0}\left(\mathfrak{g}_{C M}\right)}=\|f\|_{\mathcal{H}_{T}^{2}\left(G_{C M}\right)}=\lim _{n \rightarrow \infty}\|f\|_{L^{2}\left(G_{P_{n}}, v_{T}^{P_{n}}\right)},
$$

where $\|\cdot\|_{\mathcal{H}_{T}^{2}\left(G_{C M}\right)}$ is defined in Eq. (1.4).

Proof By Theorem 5.1 of [6], for all $P \in \operatorname{Proj}(W)$,

$$
\|f\|_{L^{2}\left(G_{P}, v_{T}^{P}\right)}=\|\hat{f}(\mathbf{e})\|_{J_{T}^{0}\left(\mathfrak{g}_{P}\right)},
$$

where

$$
\left.\|\hat{f}(\mathbf{e})\|_{J_{T}^{0}\left(\mathfrak{g}_{P}\right)}^{2}=\sum_{n=0}^{\infty} \frac{T^{n}}{n !} \sum_{\left\{h_{j}\right\}_{j=1}^{n} \subset \Gamma_{P}} \| \hat{f}(\mathbf{e}), h_{1} \otimes \cdots \otimes h_{n}\right\rangle\left.\right|^{2}
$$

and $\Gamma_{P}$ is an orthonormal basis for $\mathfrak{g}_{P}$. In particular, it follows that

$$
\|f\|_{\mathcal{H}_{T}^{2}\left(G_{C M}\right)}=\sup _{P \in \operatorname{Proj}(W)}\|\hat{f}(\mathbf{e})\|_{J_{T}^{0}\left(\mathfrak{g}_{P}\right)}
$$

and hence we must now show

$$
\sup _{P \in \operatorname{Proj}(W)}\|\hat{f}(\mathbf{e})\|_{J_{T}^{0}\left(\mathfrak{g}_{P}\right)}=\|\hat{f}(\mathbf{e})\|_{J_{T}^{0}\left(\mathfrak{g}_{C M}\right)}
$$

If $\Gamma$ is an orthonormal basis for $\mathfrak{g}_{C M}$ containing $\Gamma_{P}$, it follows that

$$
\|\hat{f}(\mathbf{e})\|_{J_{T}^{0}\left(\mathfrak{g}_{P}\right)}^{2} \leq \sum_{n=0}^{\infty} \frac{T^{n}}{n !} \sum_{\left\{h_{j}\right\}_{j=1}^{n} \subset \Gamma}\left\|\left.\left\langle\hat{f}(\mathbf{e}), h_{1} \otimes \cdots \otimes h_{n}\right\rangle\right|^{2}=\right\| \hat{f}(\mathbf{e}) \|_{J_{T}^{0}\left(\mathfrak{g}_{C M}\right)}^{2},
$$


which shows that $\sup _{P \in \operatorname{Proj}(W)}\|\hat{f}(\mathbf{e})\|_{J_{T}^{0}\left(\mathfrak{g}_{P}\right)} \leqslant\|\hat{f}(\mathbf{e})\|_{J_{T}^{0}\left(\mathfrak{g}_{C M}\right)}$. We may choose orthonormal bases, $\Gamma_{P_{n}}$, for $\mathfrak{g}_{P_{n}}$ such that $\Gamma_{P_{n}} \uparrow \Gamma$ as $n \uparrow \infty$. Then it is easy to show that

$$
\begin{aligned}
\lim _{n \rightarrow \infty}\|f\|_{L^{2}\left(G_{P_{n}}, v_{T}^{P_{n}}\right)} & =\lim _{n \rightarrow \infty}\|\hat{f}(\mathbf{e})\|_{J_{T}^{0}\left(\mathfrak{g}_{P_{n}}\right)} \\
& \left.=\lim _{n \rightarrow \infty} \sum_{n=0}^{\infty} \frac{T^{n}}{n !} \sum_{\left\{h_{j}\right\}_{j=1}^{n} \subset \Gamma_{P_{n}}} \| \hat{f}(\mathbf{e}), h_{1} \otimes \cdots \otimes h_{n}\right\rangle\left.\right|^{2} \\
& \left.=\sum_{n=0}^{\infty} \frac{T^{n}}{n !} \sum_{\left\{h_{j}\right\}_{j=1}^{n} \subset \Gamma} \| \hat{f}(\mathbf{e}), h_{1} \otimes \cdots \otimes h_{n}\right\rangle\left.\right|^{2} \\
& =\|\hat{f}(\mathbf{e})\|_{J_{T}^{0}\left(\mathfrak{g}_{C M}\right)}
\end{aligned}
$$

from which it follows that $\sup _{P \in \operatorname{Proj}(W)}\|\hat{f}(\mathbf{e})\|_{J_{T}^{0}\left(\mathfrak{g}_{P}\right)} \geqslant\|\hat{f}(\mathbf{e})\|_{J_{T}^{0} t\left(\mathfrak{g}_{C M}\right)}$.

For the next corollary, recall that $\mathcal{P}$ and $\mathcal{P}_{C M}$ denote the spaces of holomorphic cylinder polynomials on $G$ and $G_{C M}$ respectively, see Definition 1.6 and Eq. (1.7).

Corollary 6.6 If $f: G \rightarrow \mathbb{C}$ is a continuous function satisfying the bounds in Proposition 4.12 with $p=2$, then $\left.f\right|_{G_{C M}} \in \mathcal{H}_{T}^{2}\left(G_{C M}\right)$ and $\hat{f}(\mathbf{e}) \in J_{T}^{0}\left(\mathfrak{g}_{C M}\right)$. In particular, for all $T>0, \mathcal{P}_{C M} \subset \mathcal{H}_{T}^{2}\left(G_{C M}\right)$ and for any $p \in \mathcal{P}, \hat{p}(\mathbf{e}) \in J_{T}^{0}\left(\mathfrak{g}_{C M}\right)$. This shows that $\mathcal{H}_{T}^{2}\left(G_{C M}\right)$ and $J_{T}^{0}\left(\mathfrak{g}_{C M}\right)$ are non-trivial spaces.

Definition 6.7 For each $T>0$, the Taylor map is the linear map, $\mathcal{T}_{T}: \mathcal{H}_{T}^{2}\left(G_{C M}\right) \rightarrow$ $J_{T}^{0}\left(\mathfrak{g}_{C M}\right)$, defined by $\mathcal{T}_{T} f:=\hat{f}(\mathbf{e})$.

Corollary 6.8 The Taylor map, $\mathcal{T}_{T}: \mathcal{H}_{T}^{2}\left(G_{C M}\right) \rightarrow J_{T}^{0}\left(\mathfrak{g}_{C M}\right)$, is injective. Moreover, the function $\|\cdot\|_{\mathcal{H}_{T}^{2}\left(G_{C M}\right)}$ is a norm on $\mathcal{H}_{T}^{2}\left(G_{C M}\right)$ which is induced by the inner product on $\mathcal{H}_{T}^{2}\left(G_{C M}\right)$ defined by

$$
\langle u, v\rangle_{\mathcal{H}_{T}^{2}\left(G_{C M}\right)}:=\langle\hat{u}(\mathbf{e}), \hat{v}(\mathbf{e})\rangle_{J_{T}^{0}\left(\mathfrak{g}_{C M}\right)} \quad \text { for any } u, v \in \mathcal{H}_{T}^{2}\left(G_{C M}\right)
$$

Proof If $\hat{f}(\mathbf{e})=0$, then $\|f\|_{\mathcal{H}_{T}^{2}\left(G_{C M}\right)}=0$ which then implies that $\left.f\right|_{G_{P}} \equiv 0$ for all $P \in \operatorname{Proj}(W)$. As $f: G_{C M} \rightarrow \mathbb{C}$ is continuous and $\cup_{P \in \operatorname{Proj}(W)} G_{P}$ is dense in $G_{C M}$ (see the end of the proof of Theorem 5.9), it follows that $f \equiv 0$. Hence we have shown $\mathcal{T}_{T}$ injective. Since $\|\cdot\|_{J_{T}^{0}\left(\mathfrak{g}_{C M}\right)}$ is a Hilbert norm and, by Lemma 6.9, $\|f\|_{\mathcal{H}_{T}^{2}\left(G_{C M}\right)}=\left\|\mathcal{T}_{T} f\right\|_{J_{T}^{0}\left(\mathfrak{g}_{C M}\right)}$, it follows that $\|\cdot\|_{\mathcal{H}_{T}^{2}\left(G_{C M}\right)}$ is the norm on $\mathcal{H}_{T}^{2}\left(G_{C M}\right)$ induced by the inner product defined in Eq. (6.14).

Our next goal is to show that the Taylor map, $\mathcal{T}_{T}$, is surjective. The following lemma motivates the construction of the inverse of the Taylor map. 
Lemma 6.9 For every $f \in \mathcal{H}\left(G_{C M}\right)$,

$$
f(g)=\sum_{n=0}^{\infty} \frac{1}{n !}\left\langle\hat{f}_{n}(\mathbf{e}), g^{\otimes n}\right\rangle \quad \text { for any } g \in G_{C M},
$$

where the above sum is absolutely convergent. By convention, $g^{\otimes 0}=1 \in \mathbb{C}$. (For a more general version of this Lemma, see Proposition 5.1 in [3].)

Proof The function $u(z):=f(z g)$ is a holomorphic function of $z \in \mathbb{C}$. Therefore,

$$
f(g)=u(1)=\sum_{n=0}^{\infty} \frac{1}{n !} u^{(n)}(0)
$$

and the above sum is absolutely convergent. In fact, one easily sees that for all $R>0$ there exists $C(R)<\infty$ such that $\frac{1}{n !}\left|u^{(n)}(0)\right| \leqslant C(R) R^{-n}$ for all $n \in \mathbb{N}$. The proof is now completed upon observing

$$
\begin{aligned}
u^{(n)}(0) & =\left.\left(\frac{d}{d t}\right)^{n} u(t)\right|_{t=0}=\left.\left(\frac{d}{d t}\right)^{n} f(\operatorname{tg})\right|_{t=0} \\
& =\left.\left(\frac{d}{d t}\right)^{n} f\left(e^{t g}\right)\right|_{t=0}=\left(\tilde{g}^{n} f\right)(\mathbf{e})=\left\langle\hat{f}_{n}(\mathbf{e}), g^{\otimes n}\right\rangle .
\end{aligned}
$$

The next theorem is a more precise version of Theorem 1.5.

Theorem 6.10 (Taylor isomorphism theorem) For all $T>0$, the space $\mathcal{H}_{T}^{2}\left(G_{C M}\right)$ equipped with the inner product $\langle\cdot, \cdot\rangle_{\mathcal{H}_{T}^{2}\left(G_{C M}\right)}$ is a Hilbert space, $\mathcal{T}\left(\mathcal{H}_{T}^{2}\left(G_{C M}\right)\right) \subset$ $J_{T}^{0}\left(\mathfrak{g}_{C M}\right)$, and $\mathcal{T}_{T}:=\left.\mathcal{T}\right|_{\mathcal{H}_{T}^{2}\left(G_{C M}\right)}: \mathcal{H}_{T}^{2}\left(G_{C M}\right) \rightarrow J_{T}^{0}\left(\mathfrak{g}_{C M}\right)$ is a unitary transformation.

Proof Given Corollary 6.8, it only remains to prove $\mathcal{T}_{T}$ is surjective. So let $\alpha \in J_{T}^{0}\left(\mathfrak{g}_{C M}\right)$. By Lemma 6.9, if $f=\mathcal{T}_{T}^{-1} \alpha$ exists it must be given by

$$
f(g):=\sum_{n=0}^{\infty} \frac{1}{n !}\left\langle\alpha_{n}, g^{\otimes n}\right\rangle \quad \text { for any } g \in G_{C M} .
$$

We now have to check that the sum is convergent, the resulting function $f$ is in $\mathcal{H}\left(G_{C M}\right)$, and $\hat{f}(\mathbf{e})=\alpha$. Once this is done, we may apply Lemma 6.5 to conclude that $\|f\|_{\mathcal{H}_{T}^{2}\left(G_{C M}\right)}=\|\alpha\|_{J_{T}^{0}\left(\mathfrak{g}_{C M}\right)}<\infty$ and hence we will have shown that $f \in \mathcal{H}_{T}^{2}\left(G_{C M}\right)$ and $\mathcal{T}_{T} f=\alpha$. For each $n \in \mathbb{N} \cup\{0\}$, the function $u_{n}(g):=$ $\frac{1}{n !}\left\langle\alpha_{n}, g^{\otimes n}\right\rangle$ is a continuous complex $n$-linear form in $g \in G_{C M}$ and therefore holomorphic. Since $\left|\left\langle\alpha_{n}, g^{\otimes n}\right\rangle\right| \leqslant\left\|\alpha_{n}\right\|_{n}\|g\|_{\mathfrak{g}_{C M}}^{n}$, then for $R>0$

$$
\sup \left\{\left|u_{n}(g)\right|:\|g\|_{\mathfrak{g}_{C M}} \leqslant R\right\} \leqslant\left\|\alpha_{n}\right\|_{n} R^{n} .
$$


Therefore it follows that

$$
\begin{aligned}
\sum_{n=0}^{\infty} \sup \left\{\left|u_{n}(g)\right|:\|g\|_{\mathfrak{g}_{C M}} \leqslant R\right\} & \leqslant \sum_{n=0}^{\infty} \frac{T^{n}}{n !}\left\|\alpha_{n}\right\|_{n} \frac{R^{n}}{T^{n}} \\
& \leqslant \sqrt{\sum_{n=0}^{\infty} \frac{T^{n}}{n !}\left\|\alpha_{n}\right\|_{n}^{2}} \sqrt{\sum_{n=0}^{\infty} \frac{T^{n}}{n !}\left(\frac{R^{n}}{T^{n}}\right)^{2}} \\
& =\|\alpha\|_{J_{T}^{0}\left(\mathfrak{g}_{C M}\right)} e^{R^{2} /(2 T)}<\infty
\end{aligned}
$$

This shows $f(g)=\lim _{N \rightarrow \infty} \sum_{n=0}^{N} u_{n}(g)$ with the limit being uniform over $g$ in bounded subsets of $\mathfrak{g}_{C M}$. Hence, the sum in Eq. (6.16) is convergent and (see [17, Theorem 3.18.1]) the resulting function, $f$, is in $\mathcal{H}\left(G_{C M}\right)$. Since

$$
f(z h)=\sum_{n=0}^{\infty} \frac{z^{n}}{n !}\left\langle\alpha_{n}, h^{\otimes n}\right\rangle \quad \text { for any } z \in \mathbb{C} \quad \text { and } \quad h \in \mathfrak{g}_{C M},
$$

it follows that

$$
\left\langle\alpha_{n}, h^{\otimes n}\right\rangle=\left.\left(\frac{d}{d z}\right)^{n} f(z h)\right|_{z=0}=\left.\left(\frac{d}{d t}\right)^{n} f\left(e^{t h}\right)\right|_{t=0}=\left\langle\hat{f}_{n}(\mathbf{e}), h^{\otimes n}\right\rangle .
$$

This is true for all $n$ and $h \in \mathfrak{g}_{C M}$, so we may use the argument following Eq. (6.13) in [3] (or see the proof of Theorem 2.5 in [7]) to show $\hat{f}(\mathbf{e})=\alpha$.

As a consequence of Eq. (6.17) we see that if $f \in \mathcal{H}_{T}^{2}\left(G_{C M}\right)$ then

$$
|f(g)| \leqslant\|f\|_{\mathcal{H}_{T}^{2}\left(G_{C M}\right)} e^{\|g\|_{\mathfrak{g}_{C M}}^{2} /(2 T)} \quad \text { for any } g \in G_{C M}
$$

The next theorem, which is an analogue of Bargmann's pointwise bounds (see [1, Eq. (1.7)] and [6, Eq. (5.4)]), improves upon the estimate in Eq. (6.18).

Theorem 6.11 (Pointwise bounds) If $f \in \mathcal{H}_{T}^{2}\left(G_{C M}\right)$ and $g \in G_{C M}$, then for all $g \in G_{C M}$,

$$
|f(g)| \leqslant\|f\|_{\mathcal{H}_{T}^{2}\left(G_{C M}\right)} e^{d_{C M}^{2}(\mathbf{e}, g) /(2 T)}
$$

where $d_{C M}^{2}(\cdot, \cdot)$ is the distance function on $G_{C M}$ defined in Eq. (4.7).

Proof Let $P_{n} \in \operatorname{Proj}(W)$ be chosen so that $\left.P_{n}\right|_{\mathfrak{g}_{C M}} \uparrow I_{\mathfrak{g}_{C M}}$ as $n \rightarrow \infty$ and recall that $G_{0}:=\cup_{n=1}^{\infty} G_{P_{n}}$ is a dense subgroup of $G_{C M}$ as explained in the proof of Theorem 5.9. Let $g \in G_{P_{m}}$ for some $m \in \mathbb{N}$ and let $\sigma:[0,1] \rightarrow G_{C M}$ be a $C^{1}$-curve such that $\sigma(0)=\mathbf{e}$ and $\sigma(1)=g$. Then for $n \geqslant m, \sigma_{n}(t):=\pi_{P_{n}}(\sigma(t))$ is a $C^{1}$ curve in $G_{n}$ such that $\sigma_{n}(0)=\mathbf{e}$ and $\sigma_{n}(1)=g$. Therefore by [6, Eq. (5.4)], we have 


$$
|f(g)| \leqslant\left\|\left.f\right|_{G_{P_{n}}}\right\|_{L^{2}\left(G_{P_{n}}, v_{T}^{P_{n}}\right)} \cdot e^{d_{G_{P_{n}}}^{2}(\mathbf{e}, g) /(2 T)} \leqslant\|f\|_{\mathcal{H}_{T}^{2}\left(G_{C M}\right)} \cdot e^{\ell_{G_{C M}}^{2}\left(\sigma_{n}\right) /(2 T)},
$$

where $\ell_{G_{C M}}\left(\sigma_{n}\right)$ is the length of $\sigma_{n}$ as in Eq. (4.6). In the proof [4, Theorem 8.1], it was shown that $\lim _{n \rightarrow \infty} \ell_{G_{C M}}\left(\sigma_{n}\right)=\ell_{G_{C M}}(\sigma)$. Hence we may pass to the limit in Eq. (6.20) to find, $|f(g)| \leqslant\|f\|_{\mathcal{H}_{T}^{2}\left(G_{C M}\right)} \cdot e^{\ell_{G_{C M}}^{2}(\sigma) /(2 T)}$. Optimizing this last inequality over all $\sigma$ joining e to $g$ then shows that Eq. (6.19) holds for all $g \in G_{0}$. This suffices to prove Eq. (6.19) as both sides of this inequality are continuous in $g \in G_{C M}$ and $G_{0}$ is dense in $G_{C M}$.

\section{Density theorems}

The following density result is the main theorem of this section and is crucial to the next section. Techniques similar to those used in this section have appeared in Cecil [2] to prove an analogous result for path groups over stratified Lie groups.

Theorem 7.1 (Density theorem) For all $T>0, \mathcal{P}_{C M}$ defined by Eq. (1.7) is a dense subspace of $\mathcal{H}_{T}^{2}\left(G_{C M}\right)$.

Proof This theorem is a consequence of Corollary 7.4 and Proposition 7.12 below.

The remainder of this section will be devoted to proving the results used in the proof of the theorem. We will start by constructing some auxiliary dense subspaces of $J_{T}^{0}\left(\mathfrak{g}_{C M}\right)$ and $\mathcal{H}_{T}^{2}\left(G_{C M}\right)$.

\subsection{Finite rank subspaces}

Definition 7.2 A tensor, $\alpha \in J^{0}\left(\mathfrak{g}_{C M}\right)$, is said to have finite rank if $\alpha_{n}=0$ for all but finitely many $n \in \mathbb{N}$.

The next lemma is essentially a special case of [7, Lemma 3.5].

Lemma 7.3 (Finite Rank Density Lemma) The finite rank tensors in $J_{T}^{0}\left(\mathfrak{g}_{C M}\right)$ are dense in $J_{T}^{0}\left(\mathfrak{g}_{C M}\right)$.

Proof For $\theta \in \mathbb{R}$, let $\varphi_{\theta}: \mathfrak{g}_{C M} \rightarrow \mathfrak{g}_{C M}$ be defined by

$$
\varphi_{\theta}(A, a)=\left(e^{i \theta} A, e^{i 2 \theta} a\right) .
$$

Since

$$
\begin{aligned}
{\left[\varphi_{\theta}(A, a), \varphi_{\theta}(B, b)\right] } & =\left[\left(e^{i \theta} A, e^{i 2 \theta} a\right),\left(e^{i \theta} B, e^{i 2 \theta} b\right)\right] \\
& =\left(0, \omega\left(e^{i \theta} A, e^{i \theta} B\right)\right)=\left(0, e^{i 2 \theta} \omega(A, B)\right) \\
& =\varphi_{\theta}[(A, a),(B, b)]
\end{aligned}
$$

we see that $\varphi_{\theta}$ is a Lie algebra homomorphism. 
Now let $\Phi_{\theta}: \mathbf{T}\left(\mathfrak{g}_{C M}\right) \rightarrow \mathbf{T}\left(\mathfrak{g}_{C M}\right)$ be defined by $\Phi_{\theta} 1=1$ and

$$
\Phi_{\theta}\left(h_{1} \otimes \cdots \otimes h_{n}\right)=\varphi_{\theta} h_{1} \otimes \cdots \otimes \varphi_{\theta} h_{n} \quad \text { for all } h_{i} \in \mathfrak{g}_{C M} \text { and } n \in \mathbb{N}
$$

If we write $\xi \wedge \eta$ for $\xi \otimes \eta-\eta \otimes \xi$, then

$$
\begin{aligned}
\Phi_{\theta}(\xi \wedge \eta-[\xi, \eta]) & =\left(\varphi_{e^{i \theta}} \xi\right) \wedge\left(\varphi_{e^{i \theta}} \eta\right)-\varphi_{e^{i \theta}}[\xi, \eta] \\
& =\left(\varphi_{e^{i \theta}} \xi\right) \wedge\left(\varphi_{e^{i \theta}} \eta\right)-\left[\varphi_{e^{i \theta}} \xi, \varphi_{e^{i \theta}} \eta\right]
\end{aligned}
$$

From this it follows that $\Phi_{\theta}(J) \subset J$ and therefore if $\alpha \in J^{0}\left(\mathfrak{g}_{C M}\right)$, then $\alpha \circ \Phi_{\theta} \in$ $J^{0}\left(\mathfrak{g}_{C M}\right)$. Letting $\Gamma$ be an orthonormal basis as in Eq. (5.8), we have $\varphi_{\theta} h=e^{i 2 \theta} h$ or $\varphi_{\theta} h=e^{i \theta} h$ for all $h \in \Gamma$. Therefore it follows that

$$
\begin{aligned}
\left|\left\langle\alpha \circ \Phi_{\theta}, k_{1} \otimes k_{2} \otimes \cdots \otimes k_{n}\right\rangle\right|^{2} & =\left|\left\langle\alpha, \varphi_{\theta} k_{1} \otimes \varphi_{\theta} k_{2} \otimes \cdots \otimes \varphi_{\theta} k_{n}\right\rangle\right|^{2} \\
& =\left|\left\langle\alpha, k_{1} \otimes k_{2} \otimes \cdots \otimes k_{n}\right\rangle\right|^{2}
\end{aligned}
$$

and hence that

$$
\begin{aligned}
\left\|\alpha \circ \Phi_{\theta}\right\|_{J_{T}^{0}\left(\mathfrak{g}_{C M}\right)}^{2} & =\sum_{n=0}^{\infty} \frac{T^{n}}{n !} \sum_{k_{1}, k_{2}, \ldots, k_{n} \in \Gamma}\left|\left\langle\alpha \circ \Phi_{\theta}, k_{1} \otimes k_{2} \otimes \cdots \otimes k_{n}\right\rangle\right|^{2} \\
& =\sum_{n=0}^{\infty} \frac{T^{n}}{n !} \sum_{k_{1}, k_{2}, \ldots, k_{n} \in \Gamma}\left|\left\langle\alpha, k_{1} \otimes k_{2} \otimes \cdots \otimes k_{n}\right\rangle\right|^{2}=\|\alpha\|_{J_{T}^{0}\left(\mathfrak{g}_{C M}\right)}^{2}
\end{aligned}
$$

So the map $\alpha \in J_{T}^{0}\left(\mathfrak{g}_{C M}\right) \rightarrow \alpha \circ \Phi_{\theta} \in J_{T}^{0}\left(\mathfrak{g}_{C M}\right)$ is unitary. Moreover, since

$$
\begin{aligned}
& \left|\left\langle\alpha, \varphi_{\theta} k_{1} \otimes \varphi_{\theta} k_{2} \otimes \cdots \otimes \varphi_{\theta} k_{n}\right\rangle-\left\langle\alpha, k_{1} \otimes k_{2} \otimes \cdots \otimes k_{n}\right\rangle\right|^{2} \\
& \quad \leqslant 2\left|\left\langle\alpha, k_{1} \otimes k_{2} \otimes \cdots \otimes k_{n}\right\rangle\right|^{2}
\end{aligned}
$$

we may apply the dominated convergence theorem to conclude

$$
\begin{aligned}
\lim _{\theta \rightarrow 0}\left\|\alpha \circ \Phi_{\theta}-\alpha\right\|_{J_{T}^{0}\left(\mathfrak{g}_{C M}\right)}^{2} & \\
= & \sum_{n=0}^{\infty} \frac{T^{n}}{n !} \sum_{k_{1}, k_{2}, \ldots, k_{n} \in \Gamma} \lim _{\theta \rightarrow 0} \mid\left\langle\alpha, \varphi_{\theta} k_{1} \otimes \varphi_{\theta} k_{2} \otimes \cdots \otimes \varphi_{\theta} k_{n}\right\rangle \\
& -\left.\left\langle\alpha, k_{1} \otimes k_{2} \otimes \cdots \otimes k_{n}\right\rangle\right|^{2} \\
= & 0
\end{aligned}
$$

so that $\alpha \rightarrow \alpha \circ \Phi_{\theta}$ is continuous. (Notice that $\Phi_{\theta} \circ \Phi_{\alpha}=\Phi_{\theta+\alpha}$, so it suffices to check continuity at $\theta=0$.) 
Let

$$
F_{n}(\theta)=\frac{1}{2 \pi n} \sum_{k=0}^{n-1} \sum_{\ell=-k}^{k} e^{i \ell \theta}=\frac{1}{2 \pi n} \frac{\sin ^{2}(n \theta / 2)}{\sin ^{2}(\theta / 2)}
$$

denote Fejer's kernel [28, p. 143]. Then $\int_{-\pi}^{\pi} F_{n}(\theta) d \theta=1$ for all $n$ and

$$
\lim _{n \rightarrow \infty} \int_{-\pi}^{\pi} F_{n}(\theta) u(\theta) d \theta=u(0) \quad \text { for all } u \in C([-\pi, \pi], \mathbb{C})
$$

We now let

$$
\alpha(n):=\int_{-\pi}^{\pi} \alpha \circ \Phi_{\theta} F_{n}(\theta) d \theta
$$

Then

$$
\begin{aligned}
\limsup _{n \rightarrow \infty}\|\alpha-\alpha(n)\|_{J_{T}^{0}\left(\mathfrak{g}_{C M}\right)}^{2} & \leqslant \limsup _{n \rightarrow \infty}\left\|\int_{-\pi}^{\pi}\left[\alpha-\alpha \circ \Phi_{\theta}\right] F_{n}(\theta) d \theta\right\|_{J_{T}^{0}\left(\mathfrak{g}_{C M}\right)} \\
& \leqslant \limsup _{n \rightarrow \infty} \int_{-\pi}^{\pi}\left\|\alpha-\alpha \circ \Phi_{\theta}\right\|_{J_{T}^{0}\left(\mathfrak{g}_{C M}\right)} F_{n}(\theta) d \theta=0 .
\end{aligned}
$$

Moreover if $\beta:=k_{1}, \ldots, k_{m} \in \mathfrak{g}_{C M}$ with $m>n$, then there exits $\beta_{l} \in \mathfrak{g}_{C M}^{\otimes m}$ such that

$$
\Phi_{\theta} \beta=\sum_{l=m}^{2 m} e^{i l \theta} \beta_{l}
$$

From this it follows that

$$
\langle\alpha(n), \beta\rangle:=\int_{-\pi}^{\pi}\left\langle\alpha, \Phi_{\theta} \beta\right\rangle F_{n}(\theta) d \theta=\sum_{l=m}^{2 m}\left\langle\alpha, \beta_{l}\right\rangle \int_{-\pi}^{\pi} e^{i l \theta} F_{n}(\theta) d \theta=0
$$

from which it follows that $\alpha(n)_{m} \equiv 0$ for all $m>n$. Thus $\alpha(n)$ is a finite rank tensor for all $n \in \mathbb{N}$ and $\lim \sup _{n \rightarrow \infty}\|\alpha-\alpha(n)\|_{J_{T}^{0}\left(\mathfrak{g}_{C M}\right)}^{2}=0$.

Corollary 7.4 The vector space,

$$
\mathcal{H}_{T, \text { fin }}^{2}\left(G_{C M}\right):=\left\{u \in \mathcal{H}_{T}^{2}\left(G_{C M}\right): \hat{u}(\mathbf{e}) \in J_{T}^{0}\left(\mathfrak{g}_{C M}\right) \text { has finite rank }\right\}
$$

is a dense subspace of $\mathcal{H}_{T}^{2}\left(G_{C M}\right)$. 
Proof This follows directly from Lemma 7.3 and the Taylor isomorphism Theorem 6.10 .

\subsection{Polynomial approximations}

To prove Theorem 7.1, it suffices to show that every element $u \in \mathcal{H}_{T \text {, fin }}^{2}\left(G_{C M}\right)$ may be well approximated by an element from $\mathcal{H}_{T}^{2}(G)$. In order to do this, let $\left\{e_{j}: j=\right.$ $1,2,\} \subset H_{*}$ be an orthonormal basis for $H$ and for $N \in \mathbb{N}$, define $P_{N} \in \operatorname{Proj}(W)$ as in Eq. (2.17), i.e.

$$
P_{N}(w)=\sum_{j=1}^{N}\left\langle w, e_{j}\right\rangle_{H} e_{j} \quad \text { for all } w \in W
$$

Let us further define $\pi_{N}:=\pi_{P_{N}}$ and

$$
u_{N}:=u \circ \pi_{N} \quad \text { for all } N \in \mathbb{N} \text {. }
$$

We are going to prove Theorem 7.1 by showing $u_{N} \in \mathcal{P}$ and $u_{N} \rightarrow u$ in $\mathcal{H}_{T}^{2}\left(G_{C M}\right)$.

Remark 7.5 A complicating factor in showing $\left.u_{N}\right|_{G_{C M}} \rightarrow u$ in $\mathcal{H}_{T}^{2}\left(G_{C M}\right)$ is the fact that for general $\omega$ and $P \in \operatorname{Proj}(W), \pi_{P}: G \rightarrow G_{P} \subset G_{C M}$ is not a group homomorphism. In fact we have,

$$
\pi_{P}\left[(w, c) \cdot\left(w^{\prime}, c^{\prime}\right)\right]-\pi_{P}(w, c) \cdot \pi_{P}\left(w^{\prime}, c^{\prime}\right)=\Gamma_{P}\left(w, w^{\prime}\right)
$$

where

$$
\Gamma_{P}\left(w, w^{\prime}\right)=\frac{1}{2}\left(0, \omega\left(w, w^{\prime}\right)-\omega\left(P w, P w^{\prime}\right)\right)
$$

So unless $\omega$ is "supported" on the range of $P, \pi_{P}$ is not a group homomorphism. Since, $(w, b)+(0, c)=(w, b) \cdot(0, c)$ for all $w \in W$ and $b, c \in \mathbf{C}$, we may also write Eq. (7.4) as

$$
\pi_{P}\left[(w, c) \cdot\left(w^{\prime}, c^{\prime}\right)\right]=\pi_{P}(w, c) \cdot \pi_{P}\left(w^{\prime}, c^{\prime}\right) \cdot \Gamma_{P}\left(w, w^{\prime}\right) .
$$

Lemma 7.6 To each $k:=(A, a) \in \mathfrak{g}_{C M}, g=(w, c) \in G$, and $P \in \operatorname{Proj}(W)$, let

$$
k^{P}(g)=k^{P}(w, c):=\pi_{P} k+\Gamma_{P}(w, A) \in \mathfrak{g}_{P}
$$

where $\Gamma_{P}$ is defined in Eq. (7.5) above. If $u: G_{C M} \rightarrow \mathbb{C}$ is a holomorphic function and $g \in G$, then

$$
\left(\tilde{k}\left(u \circ \pi_{P}\right)\right)(g)=\left\langle D u\left(\pi_{P}(g)\right), k^{P}(g)\right\rangle
$$


or equivalently put,

$$
\left\langle\widehat{u \circ \pi_{P}}(g), k\right\rangle=\left\langle D\left(u \circ \pi_{P}\right)(g), k\right\rangle=\left\langle D u\left(\pi_{P}(g)\right), k^{P}(g)\right\rangle .
$$

Proof By direct computation,

$$
\begin{aligned}
\left(\tilde{k}\left(u \circ \pi_{P}\right)\right)(g) & =\left.\frac{d}{d t}\right|_{0} u\left(\pi_{P}\left(g \cdot e^{t k}\right)\right) \\
& =\left.\frac{d}{d t}\right|_{0}\left\langle D u\left(\pi_{P}(g)\right),\left[\pi_{P}(g)\right]^{-1} \cdot \pi_{P}\left(g \cdot e^{t k}\right)\right\rangle
\end{aligned}
$$

where by Eq. (7.6),

$$
\begin{aligned}
& \left.\frac{d}{d t}\right|_{0}\left(\left[\pi_{P}(g)\right]^{-1} \cdot \pi_{P}\left(g \cdot e^{t k}\right)\right) \\
& \quad=\left.\frac{d}{d t}\right|_{0}\left(P(t A), a+\frac{1}{2} \omega(w, t A)-\omega(P w, t P A)\right) \\
& =\left(P A, a+\frac{1}{2} \omega(w, A)-\omega(P w, P A)\right) \\
& =\pi_{P} k+\Gamma_{P}(w, A) .
\end{aligned}
$$

Notation 7.7 Given $P \in \operatorname{Proj}(W)$ and $k_{j}=\left(A_{j}, c_{j}\right) \in \mathfrak{g}_{C M}$, let $K_{j}:=k_{j}^{P}$ : $G_{C M} \rightarrow \mathfrak{g}_{C M}$ and $\kappa_{n}: G_{C M} \rightarrow \oplus_{j=1}^{n} \mathfrak{g}_{C M}^{\otimes j}$ be defined by

$$
\begin{aligned}
\kappa_{n} & =\left(\tilde{k}_{n}+K_{n} \otimes\right)\left(\tilde{k}_{n-1}+K_{n-1} \otimes\right) \cdots\left(\tilde{k}_{1}+K_{1} \otimes\right) 1 \\
& =\left(\tilde{k}_{n}+K_{n} \otimes\right)\left(\tilde{k}_{n-1}+K_{n-1} \otimes\right) \cdots\left(\tilde{k}_{2}+K_{2} \otimes\right) K_{1} .
\end{aligned}
$$

In these expressions, $K_{j} \otimes$ denotes operation of left tensor multiplication by $K_{j}$.

Example 7.8 The functions $\kappa_{n}$ are determined recursively by $\kappa_{1}=K_{1}$ and then

$$
\kappa_{n}=\left(K_{n} \otimes+\tilde{k}_{n}\right) \kappa_{n-1}=K_{n} \otimes \kappa_{n-1}+\tilde{k}_{n} \kappa_{n-1} \quad \text { for all } n \geqslant 2
$$

The first four $\kappa_{n}$ are easily seen to be given by, $\kappa_{1}=K_{1}$,

$$
\begin{aligned}
\kappa_{2} & =K_{2} \otimes K_{1}+\tilde{k}_{2} K_{1}=K_{2} \otimes K_{1}+\Gamma_{P}\left(A_{2}, A_{1}\right), \\
\kappa_{3} & =\left(K_{3} \otimes+\tilde{k}_{3}\right)\left(K_{2} \otimes K_{1}+\Gamma_{P}\left(A_{2}, A_{1}\right)\right) \\
& =K_{3} \otimes K_{2} \otimes K_{1}+K_{3} \otimes \Gamma_{P}\left(A_{2}, A_{1}\right)+\Gamma_{P}\left(A_{3}, A_{2}\right) \otimes K_{1}+K_{2} \otimes \Gamma_{P}\left(A_{3}, A_{1}\right),
\end{aligned}
$$


and

$\kappa_{4}=K_{4} \otimes K_{3} \otimes K_{2} \otimes K_{1}$ $+\left(\begin{array}{c}K_{4} \otimes K_{3} \otimes \Gamma_{P}\left(A_{2}, A_{1}\right)+K_{4} \otimes \Gamma_{P}\left(A_{3}, A_{2}\right) \otimes K_{1}+K_{4} \otimes K_{2} \otimes \Gamma_{P}\left(A_{3}, A_{1}\right) \\ +\Gamma_{P}\left(A_{4}, A_{3}\right) \otimes K_{2} \otimes K_{1}+K_{3} \otimes \Gamma_{P}\left(A_{4}, A_{2}\right) \otimes K_{1}+K_{3} \otimes K_{2} \otimes \Gamma_{P}\left(A_{4}, A_{1}\right)\end{array}\right)$
$+\Gamma_{P}\left(A_{4}, A_{3}\right) \otimes \Gamma_{P}\left(A_{2}, A_{1}\right)+\Gamma_{P}\left(A_{3}, A_{2}\right) \otimes \Gamma_{P}\left(A_{4}, A_{1}\right)+\Gamma_{P}\left(A_{4}, A_{2}\right) \otimes \Gamma_{P}\left(A_{3}, A_{1}\right)$.

At the end we will only use $\kappa_{n}$ evaluated at $\mathbf{e} \in G_{C M}$. Evaluating the above expressions at $\mathbf{e}$ amounts to replacing $K_{j}$ by $\pi_{P} k_{j}$ in all of the previous formulas.

Proposition 7.9 If $u \in \mathcal{H}\left(G_{C M}\right)$, then, with the setup in Notation 7.7, we have

$$
\left\langle\widehat{u \circ \pi_{P}}, k_{n} \otimes \cdots \otimes k_{1}\right\rangle=\left\langle\hat{u} \circ \pi_{P}, \kappa_{n}\right\rangle \quad \text { for any } n \in \mathbb{N},
$$

where both sides of this equation are holomorphic functions on $G_{C M}$.

Proof The proof is by induction with the case $n=1$ already completed via Equation (7.9). To proceed with the induction argument, suppose that Eq. (7.12) holds for some $n \in \mathbb{N}$. Then by induction and the product rule

$$
\begin{aligned}
\left\langle\widehat{u \circ \pi_{P}}, k_{n+1} \otimes k_{n} \otimes \cdots \otimes k_{1}\right\rangle & =\tilde{k}_{n+1}\left\langle\widehat{u \circ \pi_{P}}, k_{n+1} \otimes k_{n} \otimes \cdots \otimes k_{1}\right\rangle \\
& =\tilde{k}_{n+1}\left\langle\hat{u} \circ \pi_{P}, \kappa_{n}\right\rangle \\
& =\left\langle\hat{u} \circ \pi_{P}, \tilde{k}_{n+1} \kappa_{n}\right\rangle+\left\langle\tilde{k}_{n+1}\left[\hat{u} \circ \pi_{P}\right], \kappa_{n}\right\rangle .
\end{aligned}
$$

To evaluate $\tilde{k}_{n+1}\left[\hat{u} \circ \pi_{P}\right]$ let $v \in \mathbf{T}\left(\mathfrak{g}_{C M}\right)$ and let $\tilde{v}$ denote the corresponding left invariant differential operator on $G_{C M}$. Then

$$
\begin{aligned}
& \left\langle\tilde{k}_{n+1}\left[\hat{u} \circ \pi_{P}\right], v\right\rangle(g)=\left(\tilde{k}_{n+1}\left\langle\left[\hat{u} \circ \pi_{P}\right], v\right\rangle\right)(g) \\
& =\left(\tilde{k}_{n+1}\left[(\tilde{v} u) \circ \pi_{P}\right]\right)(g) \\
& =\left\langle D(\tilde{v} u)\left(\pi_{P}(g)\right), k_{n+1}^{P}(g)\right\rangle \\
& =\left(\widetilde{k_{n+1}^{P}(g)} \tilde{v} u\right)\left(\pi_{P}(g)\right) \\
& =\left\langle\hat{u}\left(\pi_{P}(g)\right), k_{n+1}^{P}(g) \otimes v\right\rangle \text {. }
\end{aligned}
$$

Combining Eqs. (7.13) and (7.14) shows,

$$
\begin{aligned}
\left\langle\widehat{u \circ \pi_{P}}, k_{n+1} \otimes k_{n} \otimes \cdots \otimes k_{1}\right\rangle & =\left\langle\hat{u} \circ \pi_{P}, \tilde{k}_{n+1} \kappa_{n}\right\rangle+\left\langle\hat{u} \circ \pi_{P}, k_{n+1}^{P} \otimes \kappa_{n}\right\rangle \\
& =\left\langle\hat{u} \circ \pi_{P}, \tilde{k}_{n+1} \kappa_{n}+k_{n+1}^{P} \otimes \kappa_{n}\right\rangle=\left\langle\hat{u} \circ \pi_{P}, \kappa_{n+1}\right\rangle
\end{aligned}
$$

wherein we have used Eq. (7.11) for the last equality. 
The induction proof of the following lemma will be left to the reader with Example 7.8 as a guide.

Lemma 7.10 Let $k_{j}=\left(A_{j}, c_{j}\right) \in \mathfrak{g}_{C M}$ for $1 \leqslant j \leqslant n,\left\lfloor\frac{n}{2}\right\rfloor=n / 2$ if $n$ is even and $(n-1) / 2$ if $n$ is odd, and $\kappa_{n}$ be as in Eq. (7.10). Then

$$
\kappa_{n}(\mathbf{e})=\pi_{P} k_{n} \otimes \cdots \otimes \pi_{P} k_{2} \otimes \pi_{P} k_{1}+R\left(P: k_{n}, \ldots, k_{1}\right),
$$

where

$$
R\left(P: k_{n}, \ldots, k_{1}\right)=\sum_{j=1}^{\left\lfloor\frac{n}{2}\right\rfloor} R_{j}\left(P: k_{n}, \ldots, k_{1}\right)
$$

with $R_{j}\left(P: k_{1}, \ldots, k_{n}\right) \in \mathfrak{g}_{C M}^{\otimes(n-j)}$. Each remainder term, $R_{j}\left(P: k_{1}, \ldots, k_{n}\right)$, is a linear combination (with coefficients coming from $\{ \pm 1,0\}$ ) of homogenous tensors which are permutations of the indices and order of the terms in the tensor product of the form

$$
\Gamma_{P}\left(A_{1}, A_{2}\right) \otimes \cdots \otimes \Gamma_{P}\left(A_{2 j-1}, A_{2 j}\right) \otimes k_{2 j+1} \otimes \cdots \otimes k_{n}
$$

Proposition 7.11 Let $P_{N} \in \operatorname{Proj}(W)$ and $\pi_{N}:=\pi_{P_{N}}$ be as in Notation 1.1 and suppose that $u \in \mathcal{H}\left(G_{C M}\right)$ satisfies $\left\|\hat{u}_{n}(\mathbf{e})\right\|_{n}<\infty$ for all $n$. Then

$$
\lim _{N \rightarrow \infty}\left\|\hat{u}_{n}(\mathbf{e})-\left[\widehat{u \circ \pi_{N}}(\mathbf{e})\right]_{n}\right\|_{n}=0 \quad \text { for } n=0,1,2, \cdots
$$

Proof To simplify notation, let $\alpha_{n}:=\hat{u}_{n}(\mathbf{e})$ and $\alpha_{n}(N):=\left[\widehat{u \circ \pi_{N}}(\mathbf{e})\right]_{n}$. Let $\Gamma$ be an orthonormal basis for $\mathfrak{g}_{C M}$ of the form in Eq. (5.8) and let $\mathbf{k}:=\left(k_{1}, k_{2}, \ldots, k_{n}\right) \in \Gamma^{n}$. Then

$$
\begin{aligned}
\left\langle\alpha-\alpha(N), k_{1} \otimes \cdots \otimes k_{n}\right\rangle= & \left\langle\alpha, k_{1} \otimes \cdots \otimes k_{n}-\pi_{N} k_{1} \otimes \cdots \otimes \pi_{N} k_{n}\right\rangle \\
& +\left\langle\alpha, R\left(P_{N}: \mathbf{k}\right)\right\rangle
\end{aligned}
$$

where $R\left(P_{N}: \mathbf{k}\right)$ is as in Lemma 7.10. Therefore, $\left\|\alpha_{n}-\alpha_{n}(N)\right\|_{n} \leqslant C_{N}+D_{N}$ where

$$
\begin{aligned}
C_{N} & :=\sqrt{\sum_{\mathbf{k} \in \Gamma^{n}}\left|\left\langle\alpha, R\left(P_{N}: \mathbf{k}\right)\right\rangle\right|^{2}} \text { and } \\
D_{N} & :=\sqrt{\sum_{\mathbf{k} \in \Gamma^{n}}\left|\left\langle\alpha_{n}, k_{1} \otimes \cdots \otimes k_{n}-\pi_{N} k_{1} \otimes \cdots \otimes \pi_{N} k_{n}\right\rangle\right|^{2}} .
\end{aligned}
$$


We will complete the proof by showing that, $\lim _{N \rightarrow \infty} C_{N}=0=\lim _{N \rightarrow \infty} D_{N}$. To estimate $C_{N}$, use Lemma 7.10 and the triangle inequality for $\ell_{2}\left(\Gamma^{n}\right)$ to find,

$$
C_{N}=\sqrt{\sum_{\mathbf{k} \in \Gamma^{n}}\left|\sum_{j=1}^{\left\lfloor\frac{n}{2}\right\rfloor}\left\langle\alpha, R_{j}\left(P_{N}: \mathbf{k}\right)\right\rangle\right|^{2}} \leqslant \sum_{j=1}^{\left\lfloor\frac{n}{2}\right\rfloor} \sqrt{\sum_{\mathbf{k} \in \Gamma^{n}}\left|\left\langle\alpha, R_{j}\left(P_{N}: \mathbf{k}\right)\right\rangle\right|^{2}} .
$$

But $\sum_{\mathbf{k} \in \Gamma^{n}}\left|\left\langle\alpha, R_{j}\left(P_{N}: \mathbf{k}\right)\right\rangle\right|^{2}$ is bounded by a sum of terms (the number of these terms depends only on $j$ and $n$ and not $N$ ) of which a typical term (see Eq. (7.17)) is;

$$
\sum_{\mathbf{k} \in \Gamma^{n}}\left|\left\langle\alpha_{n-j}, \Gamma_{P_{N}}\left(A_{1}, A_{2}\right) \otimes \cdots \otimes \Gamma_{P_{N}}\left(A_{2 j-1}, A_{2 j}\right) \otimes k_{2 j+1} \otimes \cdots \otimes k_{n}\right\rangle\right|^{2} .
$$

The sum in Eq. (7.19) may be estimated by,

$$
\left\|\alpha_{n-j}\right\|_{n-j} \sum_{l_{1}, \ldots, l_{2 j}=1}^{\infty}\left\|\Gamma_{P_{N}}\left(e_{l_{1}}, e_{l_{2}}\right)\right\|_{\mathfrak{g}_{C M}}^{2} \ldots\left\|\Gamma_{P_{N}}\left(e_{l_{2 j-1}}, e_{l_{2 j}}\right)\right\|_{\mathfrak{g}_{C M}}^{2}=\left\|\alpha_{n-j}\right\|_{n-j}^{2} \varepsilon_{N}^{j}
$$

where

$$
\begin{aligned}
\varepsilon_{N} & =\frac{1}{4} \sum_{k, l=1}^{\infty}\left\|\omega\left(e_{k}, e_{l}\right)-\omega\left(P_{N} e_{k}, P_{N} e_{l}\right)\right\|_{\mathbf{C}}^{2} \\
& =\frac{1}{4} \sum_{\max (k, l)>N}^{\infty}\left\|\omega\left(e_{k}, e_{l}\right)-\omega\left(P_{N} e_{k}, P_{N} e_{l}\right)\right\|_{\mathbf{C}}^{2} \\
& \leqslant \frac{1}{2} \sum_{\max (k, l)>N}^{\infty}\left\|\omega\left(e_{k}, e_{l}\right)\right\|_{\mathbf{C}}^{2} \rightarrow 0 \text { and } N \rightarrow \infty .
\end{aligned}
$$

Thus we have shown $\lim _{N \rightarrow \infty} C_{N}=0$

For $N \in \mathbb{N}$, let $\Gamma_{N}=\left\{\left(0, f_{j}\right)\right\}_{j=1}^{d} \cup\left\{\left(e_{j}, 0\right)\right\}_{j=1}^{N}$. Since $k_{1} \otimes \cdots \otimes k_{n}=\pi_{N} k_{1}$ $\otimes \cdots \otimes \pi_{N} k_{n}$ if $\mathbf{k}:=\left(k_{1}, k_{2}, \ldots, k_{n}\right) \in \Gamma_{N}^{n}$, it follows that

$$
\begin{aligned}
D_{N}^{2} & =\sum_{\mathbf{k} \in \Gamma^{n} \backslash \Gamma_{N}^{n}}\left|\left\langle\alpha_{n}, k_{1} \otimes \cdots \otimes k_{n}-\pi_{N} k_{1} \otimes \cdots \otimes \pi_{N} k_{n}\right\rangle\right|^{2} \\
& \leqslant 2 \sum_{\mathbf{k} \in \Gamma^{n} \backslash \Gamma_{N}^{n}}\left|\left\langle\alpha_{n}, k_{1} \otimes \cdots \otimes k_{n}\right\rangle\right|^{2}
\end{aligned}
$$

Because

$$
\sum_{\mathbf{k} \in \Gamma^{n}}\left|\left\langle\alpha_{n}, k_{1} \otimes \cdots \otimes k_{n}\right\rangle\right|^{2}=\left\|\alpha_{n}\right\|_{n}^{2}<\infty
$$


and $\Gamma_{N}^{n} \uparrow \Gamma_{N}$ as $N \uparrow \infty$, the sum in Eq. (7.20) tends to zero as $N \rightarrow \infty$. Thus $\lim _{N \rightarrow \infty} D_{N}=0$ and the proof is complete.

Proposition 7.12 If $u \in \mathcal{H}_{T, \text { fin }}^{2}\left(G_{C M}\right)$ and $u_{N}:=u \circ \pi_{N}$ as in Eq. (7.3), then $u_{N} \in \mathcal{P}$ and $\left.u_{N}\right|_{G_{C M}} \rightarrow u$ in $\mathcal{H}_{T}^{2}\left(G_{C M}\right)$.

Proof Suppose $m \in \mathbb{N}$ is chosen so that $\hat{u}_{n}(\mathbf{e})=0$ if $n>m$. According to Proposition 7.9,

$$
\left\langle\hat{u}_{N}(\mathbf{e}), k_{n} \otimes \cdots \otimes k_{1}\right\rangle=\left\langle\hat{u}(\mathbf{e}), \kappa_{n}(\mathbf{e})\right\rangle
$$

where $\kappa_{n}(\mathbf{e}) \in \bigoplus_{j=1}^{\left\lfloor\frac{n}{2}\right\rfloor} \mathfrak{g}_{C M}^{\otimes(n-j)}$. From this it follows that $\left\langle\hat{u}_{N}(\mathbf{e}), k_{n} \otimes \cdots \otimes k_{1}\right\rangle=0$ if $n \geqslant 2 m+2$. Therefore, $u_{N}$ restricted to $P_{N} H \times \mathbf{C}$ is a holomorphic polynomial and since $u_{N}=\left.u_{N}\right|_{P_{N} H \times \mathbf{C}} \circ \pi_{N}$, it follows that $u_{N} \in \mathcal{P}$. Moreover,

$$
\lim _{N \rightarrow \infty}\left\|\hat{u}(\mathbf{e})-\hat{u}_{N}(\mathbf{e})\right\|_{J_{T}^{0}\left(\mathfrak{g}_{C M}\right)}^{2}=\lim _{N \rightarrow \infty} \sum_{n=0}^{2 m+2} \frac{T^{n}}{n !}\left\|\hat{u}_{n}(\mathbf{e})-\left[\hat{u}_{N}(\mathbf{e})\right]_{n}\right\|_{n}^{2}=0
$$

wherein we have used Proposition 7.11 to conclude $\lim _{N \rightarrow \infty}\left\|\hat{u}_{n}(\mathbf{e})-\left[\hat{u}_{N}(\mathbf{e})\right]_{n}\right\|_{n}=0$ for all $n$. It then follows by the Taylor isomorphism Theorem 6.10 that $\lim _{N \rightarrow \infty} \| u-$ $u_{N} \|_{\mathcal{H}_{T}^{2}\left(G_{C M}\right)}=0$.

\section{The skeleton isomorphism}

This section is devoted to the proof of the skeleton Theorem 1.8. Let us begin by gathering together a couple of results that we have already proved.

Proposition 8.1 If $f: G \rightarrow \mathbb{C}$ is a continuous function such that $\left.f\right|_{G_{C M}}$ is holomorphic, then

$$
\|f\|_{L^{2}\left(v_{T}\right)} \leqslant\left\|\left.f\right|_{G_{C M}}\right\|_{\mathcal{H}_{T}^{2}\left(G_{C M}\right)}=\|\hat{f}(\mathbf{e})\|_{J_{T}^{0}\left(\mathfrak{g}_{C M}\right)} .
$$

If $\left\|\left.f\right|_{G_{C M}}\right\|_{\mathcal{H}_{T}^{2}\left(G_{C M}\right)}^{2}<\infty$, then $S_{T} f=f$ and $f$ satisfies the Gaussian pointwise bounds in Eq. (6.19). (See Corollary 8.3 for a more sophisticated version of this proposition.)

Proof See Theorems 5.9 and 6.11.

Lemma 8.2 Let $f: G \rightarrow \mathbb{C}$ be a continuous function such that $\left.f\right|_{G_{C M}}$ is holomorphic and let $\delta>0$ be as in Theorem 4.11. If there exists an $\varepsilon \in(0, \delta)$ such that $|f(\cdot)| \leqslant C e^{\varepsilon \rho^{2}(\cdot) /(2 T)}$ on $G$, then

$$
\|f\|_{L^{2}\left(v_{T}\right)}=\|f\|_{\mathcal{H}_{T}^{2}\left(G_{C M}\right)}=\|\hat{f}(\mathbf{e})\|_{J_{T}^{0}\left(\mathfrak{g}_{C M}\right)}<\infty
$$

(It will be shown in Corollary 8.4 that $f$ is actually in $\mathcal{H}_{T}^{2}(G)$.) In particular, Eq. (8.2) holds for all $f \in \mathcal{P}$. 
Proof Let $\left\{P_{n}\right\}_{n=1}^{\infty} \subset \operatorname{Proj}(W)$ be a sequence such that $\left.P_{n}\right|_{\mathfrak{g}_{C M}} \uparrow I_{\mathfrak{g}_{C M}}$ as $n \rightarrow \infty$. Then, by Lemma 6.5 and Proposition 4.12 with $h=0$,

$$
\infty>\|f\|_{L^{2}\left(v_{T}\right)}=\lim _{n \rightarrow \infty}\|f\|_{L^{2}\left(G_{P_{n}} v_{T}^{P_{n}}\right)}=\|f\|_{\mathcal{H}_{T}^{2}\left(G_{C M}\right)}=\|\hat{f}(\mathbf{e})\|_{J_{T}^{0}\left(\mathfrak{g}_{C M}\right)} .
$$

We are now ready to complete the proof of the Skeleton isomorphism Theorem 1.8.

\subsection{Proof of Theorem 1.8}

Proof By Corollary 5.10, $S_{T} f=\left.f\right|_{G_{C M}}$ for all $f \in \mathcal{P}$ and hence by Lemma 8.2, $\left\|S_{T} f\right\|_{\mathcal{H}_{T}^{2}\left(G_{C M}\right)}=\|f\|_{L^{2}\left(v_{T}\right)}$. It therefore follows that $\left.S_{T}\right|_{\mathcal{P}}$ extends uniquely to an isometry, $\bar{S}_{T}$, from $\mathcal{H}_{T}^{2}(G)$ to $\mathcal{H}_{T}^{2}\left(G_{C M}\right)$ such that $\bar{S}_{T}(\mathcal{P})=\mathcal{P}_{C M}$. Since $\bar{S}_{T}$ is isometric and $\mathcal{P}_{C M}$ is dense in $\mathcal{H}_{T}^{2}\left(G_{C M}\right)$, it follows that $\bar{S}_{T}$ is surjective, i.e. $\bar{S}_{T}$ : $\mathcal{H}_{T}^{2}(G) \rightarrow \mathcal{H}_{T}^{2}\left(G_{C M}\right)$ is a unitary map. To finish the proof we only need to show $S_{T} f=\bar{S}_{T} f$ for all $f \in \mathcal{H}_{T}^{2}(G)$. Let $p_{n} \in \mathcal{P}$ such that $p_{n} \rightarrow f$ in $L^{2}\left(v_{T}\right)$. Then $p_{n}=S_{T} p_{n} \rightarrow \bar{S}_{T} f$ in $\mathcal{H}_{T}^{2}\left(G_{C M}\right)$ and hence by the Gaussian pointwise bounds in Eq. (6.19), $\bar{S}_{T} f(g)=\lim _{n \rightarrow \infty} p_{n}(g)$ for all $g \in G_{C M}$. Similarly, using the Gaussian bounds in Corollary 4.8 , it follows that

$$
\begin{aligned}
\left|S_{T} f(g)-p_{n}(g)\right| & =\left|S_{T}\left(f-p_{n}\right)(g)\right| \\
& \leqslant\left\|f-p_{n}\right\|_{L^{2}\left(v_{T}\right)} \exp \left(\frac{c(k(\omega) T / 2)}{T} d_{G_{C M}}^{2}(\mathbf{e}, g)\right)
\end{aligned}
$$

and hence we also have, $S_{T} f(g)=\lim _{n \rightarrow \infty} p_{n}(g)$ for all $g \in G_{C M}$. Therefore, $S_{T} f=\bar{S}_{T} f$ as was to be shown.

Corollary 8.3 If $f: G \rightarrow \mathbb{C}$ is a continuous function such that $\left.f\right|_{G_{C M}} \in \mathcal{H}_{T}^{2}\left(G_{C M}\right)$, then $f \in \mathcal{H}_{T}^{2}(G), S_{T} f=\left.f\right|_{G_{C M}}$, and $\|f\|_{L^{2}\left(v_{T}\right)}=\|f\|_{\mathcal{H}_{T}^{2}\left(G_{C M}\right)}$.

Proof By Proposition 8.1 we already know that $S_{T} f=\left.f\right|_{G_{C M}}$. By Theorem 1.8, there exists $u \in \mathcal{H}_{T}^{2}(G)$ such that $\left.f\right|_{G_{C M}}=S_{T} u$. Let $p_{n} \in \mathcal{P}$ be chosen so that $p_{n} \rightarrow u$ in $L^{2}\left(v_{T}\right)$ and hence $\left.p_{n}\right|_{G_{C M}}=S_{T} p_{n} \rightarrow S_{T} u=S_{T} f$ in $\mathcal{H}_{T}^{2}\left(G_{C M}\right)$ as $n \rightarrow \infty$. Hence it follows from Proposition 8.1 that

$$
\left\|f-p_{n}\right\|_{L^{2}\left(v_{T}\right)} \leqslant\left\|\left.\left(f-p_{n}\right)\right|_{G_{C M}}\right\|_{\mathcal{H}_{T}^{2}\left(G_{C M}\right)}=\left\|S_{T}\left(f-p_{n}\right)\right\|_{\mathcal{H}_{T}^{2}\left(G_{C M}\right)},
$$

and therefore, $\lim _{n \rightarrow \infty}\left\|f-p_{n}\right\|_{L^{2}\left(v_{T}\right)}=0$, i.e. $p_{n} \rightarrow f$ in $L^{2}\left(v_{T}\right)$. Since $p_{n} \rightarrow u$ in $L^{2}\left(v_{T}\right)$ as well, we may conclude that $f=u \in \mathcal{H}_{T}^{2}(G)$.

Corollary 8.4 Suppose that $f: G \rightarrow \mathbb{C}$ is a continuous function such that $|f| \leqslant$ $C e^{\varepsilon \rho^{2} /(2 T)}$ and $\left.f\right|_{G_{C M}}$ is holomorphic, then $f \in \mathcal{H}_{T}^{2}(G)$ and $S_{T} f=f$.

Proof This is a consequence of Lemma 8.2 and Corollary 8.3. 


\section{The holomorphic chaos expansion}

This section is devoted to the proof of the holomorphic chaos expansion Theorem 1.9 (or equivalently Theorem 9.10). Before going to the proof we will develop the machinery necessary in order to properly define the right side of Eq. (1.8).

9.1 Generalities about multiple Itô integrals

Let $(\mathbb{H}, \mathbb{W})$ be a complex abstract Wiener space. Analogous to the notation used in Sect. 6.1, we will denote the norm on $\mathbb{H}^{* \otimes n}$ by $\|\cdot\|_{n}$.

Notation 9.1 For $\alpha \in \mathbb{H}^{* \otimes n}$ and $P \in \operatorname{Proj}(\mathbb{W})$, let $\alpha_{P}:=\alpha \circ P^{\otimes n} \in \mathbb{H}^{* \otimes n}$.

Proposition 9.2 Let $n \in \mathbb{N}$ and $\alpha \in \mathbb{H}^{* \otimes n}$ and $P_{k} \in \operatorname{Proj}(\mathbb{W})$ with $\left.\left.P_{k}\right|_{\mathbb{H}} \uparrow I\right|_{\mathbb{H} \text {. Then }}$ $\alpha_{P_{k}} \rightarrow \alpha$ in $\mathbb{H}^{* \otimes n}$.

Proof Let $\Lambda:=\cup_{k} \Lambda_{k}$ be an orthonormal basis for $\mathbb{H}$ where $\Lambda_{k}$ is chosen to be an orthonormal basis for $\operatorname{Ran}\left(P_{k}\right)$ such that $\Lambda_{k} \subset \Lambda_{k+1}$ for all $k$. Since $P_{k} u=u$ or $P_{k} u=0$ for all $u \in \Lambda$ and $k \in \mathbb{N}$, we have

$$
\left|\left\langle\alpha, u_{1} \otimes \cdots \otimes u_{n}-P_{k} u_{1} \otimes \cdots \otimes P_{k} u_{n}\right\rangle\right|^{2} \leq\left|\left\langle\alpha, u_{1} \otimes \cdots \otimes u_{n}\right\rangle\right|^{2}
$$

where

$$
\sum_{u_{1}, \ldots, u_{n} \in \Lambda}\left|\left\langle\alpha, u_{1} \otimes \cdots \otimes u_{n}\right\rangle\right|^{2}=\|\alpha\|_{n}^{2}<\infty .
$$

An application of the dominated convergence theorem then implies,

$$
\begin{aligned}
\lim _{k \rightarrow \infty}\left\|\alpha-\alpha_{P_{k}}\right\|_{n}^{2} & =\lim _{k \rightarrow \infty} \sum_{u_{1}, \ldots, u_{n} \in \Lambda}\left|\left\langle\alpha, u_{1} \otimes \cdots \otimes u_{n}-P_{k} u_{1} \otimes \cdots \otimes P_{k} u_{n}\right\rangle\right|^{2} \\
& =\sum_{u_{1}, \ldots, u_{n} \in \Lambda} \lim _{k \rightarrow \infty}\left|\left\langle\alpha, u_{1} \otimes \cdots \otimes u_{n}-P_{k} u_{1} \otimes \cdots \otimes P_{k} u_{n}\right\rangle\right|^{2}=0
\end{aligned}
$$

Lemma 9.3 Suppose that $\{b(t)\}_{t \geq 0}$ is a $\mathbb{W}$-valued Brownian motion normalized by

$$
\mathbb{E}\left[\ell_{1}(b(t)) \ell_{2}(b(s))\right]=\frac{1}{2} s \wedge t\left(\ell_{1}, \ell_{2}\right)_{\mathbb{H}_{\mathrm{Re}}^{*}} \text { for all } \ell_{1}, \ell_{2} \in \mathbb{W}_{\mathrm{Re}}^{*}
$$

If $P \in \operatorname{Proj}(\mathbb{W}), T>0$, and $\left\{f_{s}\right\}_{s \geq 0}$ is a $(P \mathbb{H})^{*}$-valued continuous adapted process, such that $\mathbb{E} \int_{0}^{T}\left|f_{s}\right|_{(P \mathbb{H})^{*}}^{2} d s<\infty$, then

$$
\mathbb{E}\left|\int_{0}^{T}\left\langle f_{s}, d(P b)(s)\right\rangle\right|^{2}=\int_{0}^{T} \mathbb{E}\left|f_{s}\right|_{(P \mathbb{H})^{*}}^{2} d s .
$$


Proof Let $\left\{e_{j}\right\}_{j=1}^{d}$ be an orthonormal basis for $P \mathbb{H}$ and write

$$
P b(s)=\sum_{j=1}^{d}\left[X_{j}(s) e_{j}+Y_{j}(s) i e_{j}\right]
$$

where $X_{j}(s)=\operatorname{Re}\left(P b(s), e_{j}\right)$ and $Y_{j}(s)=\operatorname{Im}\left(P b(s), e_{j}\right)$. From the normalization in Eq. (9.1) it follows that $\left\{\sqrt{2} X_{j}, \sqrt{2} Y_{j}\right\}_{j=1}^{d}$ is a sequence of independent standard Brownian motions, and therefore the quadratic covariations of these processes are given by:

$d X_{j} d Y_{k}=0 \quad$ and $\quad d X_{j} d X_{k}=d Y_{j} d Y_{k}=\frac{1}{2} \delta_{j k} d t \quad$ for all $j, k=1, \ldots, d$

Using Eq. (9.3) along with the identity,

$$
\left\langle f_{s}, d(P b)(s)\right\rangle=\sum_{j=1}^{d}\left[\left\langle f_{s}, e_{j}\right\rangle d X_{j}(s)+\left\langle f_{s}, i e_{j}\right\rangle d Y_{j}(s)\right]
$$

it follows by the basic isometry property of the stochastic integral that

$$
\begin{aligned}
\mathbb{E}\left|\int_{0}^{T}\left\langle f_{s}, d(P b)(s)\right\rangle\right|^{2} & =\frac{1}{2} \sum_{j=1}^{d} \mathbb{E}\left[\int_{0}^{T}\left|\left\langle f_{s}, e_{j}\right\rangle\right|^{2} d s+\int_{0}^{T}\left|\left\langle f_{s}, i e_{j}\right\rangle\right|^{2} d s\right] \\
& =\mathbb{E} \int_{0}^{T} \sum_{j=1}^{d}\left|\left\langle f_{s}, e_{j}\right\rangle\right|^{2} d s=\int_{0}^{T} \mathbb{E}\left|f_{s}\right|_{(P \mathbb{H})^{*}}^{2} d s .
\end{aligned}
$$

Definition 9.4 For $P \in \operatorname{Proj}(\mathbb{W}), n \in \mathbb{N}$, and $T>0$, let

$$
M_{n}^{P}(T):=\int_{0 \leq s_{1} \leq s_{2} \leq \cdots \leq s_{n} \leq T} d P b\left(s_{1}\right) \otimes d P b\left(s_{2}\right) \otimes \cdots \otimes d P b\left(s_{n}\right) .
$$

Alternatively put, $M_{0}^{P}(T) \equiv 1$ and $M_{n}^{P}(t) \in(P \mathbb{H})^{\otimes n}$ is defined inductively by

$$
M_{n}^{P}(t):=\int_{0}^{t} M_{n-1}^{P}(s) \otimes d P b(s) \quad \text { for all } t \geq 0
$$


Corollary 9.5 Suppose that $T>0, \alpha \in \mathbb{H}^{* \otimes n}$, and $P \in \operatorname{Proj}(\mathbb{W})$, then $\left\langle\alpha, M_{n}^{P}(T)\right\rangle$ is a square integrable random variable and

$$
\mathbb{E}\left|\left\langle\alpha, M_{n}^{P}(T)\right\rangle\right|^{2}=\frac{T^{n}}{n !}\left\|\alpha_{P}\right\|_{n}^{2} .
$$

Proof The proof is easily carried out by induction with the case $n=1$ following directly from Lemma 9.3. Similarly from Lemma 9.3, Eq. (9.5), and induction we have

$$
\begin{aligned}
\mathbb{E}\left|\left\langle\alpha, M_{n}^{P}(T)\right\rangle\right|^{2} & =\mathbb{E}\left|\int_{0}^{T}\left\langle\alpha, M_{n-1}^{P}(s) \otimes d P b(s)\right\rangle\right|^{2} \\
& =\int_{0}^{T} \sum_{j=1}^{d} \mathbb{E}\left|\left\langle\alpha, M_{n-1}^{P}(s) \otimes e_{j}\right\rangle\right|^{2} d s \\
& =\sum_{j=1}^{d} \int_{0}^{T} \frac{s^{n-1}}{(n-1) !}\left\|\left\langle\alpha,(\cdot) \otimes e_{j}\right\rangle\right\|_{n-1}^{2} d s=\frac{T^{n}}{n !}\left\|\alpha_{p}\right\|_{n}^{2} .
\end{aligned}
$$

Notation 9.6 We now fix $T>0$ and for $P \in \operatorname{Proj}(\mathbb{W})$, let $\tilde{\alpha}_{P}=\left\langle\alpha, M_{n}^{P}(T)\right\rangle$, i.e.

$$
\tilde{\alpha}_{P}=\left\langle\alpha, \int_{0 \leq s_{1} \leq s_{2} \leq \cdots \leq s_{n} \leq T} d P b\left(s_{1}\right) \otimes d P b\left(s_{2}\right) \otimes \cdots \otimes d P b\left(s_{n}\right)\right\rangle .
$$

Lemma 9.7 If $P, Q \in \operatorname{Proj}(\mathbb{W})$, then

$$
\left\|\tilde{\alpha}_{P}-\tilde{\alpha}_{Q}\right\|_{L^{2}}^{2}:=\mathbb{E}\left|\tilde{\alpha}_{P}-\tilde{\alpha}_{Q}\right|^{2}=\frac{T^{n}}{n !}\left\|\alpha_{P}-\alpha_{Q}\right\|_{n}^{2} .
$$

Proof Let $R \in \operatorname{Proj}(\mathbb{W})$ be the orthogonal projection onto $\operatorname{Ran}(P)+\operatorname{Ran}(Q)$. We then have $\left(\alpha_{P}\right)_{R}=\alpha_{P}$ and $\left(\alpha_{Q}\right)_{R}=\alpha_{Q}$ and therefore, by Corollary 9.5,

$$
\begin{aligned}
\mathbb{E}\left|\tilde{\alpha}_{P}-\tilde{\alpha}_{Q}\right|^{2} & =\mathbb{E} \mid\left(\alpha_{P}{\tilde{)_{R}}}-\left.\left(\alpha_{Q}\right)_{R}\right|^{2}=\mathbb{E}\left|\left(\alpha_{P}-\alpha_{Q}\right)_{R}\right|^{2}\right. \\
& =\frac{T^{n}}{n !}\left\|\left(\alpha_{P}-\alpha_{Q}\right)_{R}\right\|_{n}^{2}=\frac{T^{n}}{n !}\left\|\alpha_{P}-\alpha_{Q}\right\|_{n}^{2} .
\end{aligned}
$$

Proposition 9.8 Let $\alpha \in \mathbb{H}^{* \otimes n}$ and $P_{k} \in \operatorname{Proj}(\mathbb{W})$ with $\left.\left.P_{k}\right|_{\mathbb{H}} \uparrow I\right|_{\mathbb{H}}$, then $\left\{\tilde{\alpha}_{P_{k}}\right\}_{k=1}^{\infty}$ is an $L^{2}$-convergent series. We denote the limit by $\tilde{\alpha}$. This limit is independent of the choice of orthogonal projections used in constructing $\tilde{\alpha}$. 
Proof For $k, l \in \mathbb{N}$, by Lemma 9.7,

$$
\left\|\tilde{\alpha}_{P_{l}}-\tilde{\alpha}_{P_{k}}\right\|_{L^{2}}=\left\|\alpha_{P_{l}}-\alpha_{P_{k}}\right\|_{n} \rightarrow 0 \text { as } l, k \rightarrow \infty,
$$

because, as we have already seen, $\alpha_{P_{l}} \rightarrow \alpha$ in $\mathbb{H}^{* \otimes n}$. Therefore $\tilde{\alpha}:=L^{2}-\lim _{k \rightarrow \infty} \tilde{\alpha}_{P_{k}}$ exists.

Now suppose that $Q_{l} \in \operatorname{Proj}(\mathbb{W})$ also increases to $\left.I\right|_{\mathbb{H}}$. By Lemma 9.7 and the fact that both $\alpha_{P_{l}}$ and $\alpha_{Q_{l}}$ converge to $\alpha$ in $\mathbb{H}^{* \otimes n}$, we have

$$
\left\|\tilde{\alpha}_{P_{l}}-\tilde{\alpha}_{Q_{l}}\right\|_{L^{2}}=\left\|\alpha_{P_{l}}-\alpha_{Q_{l}}\right\|_{\mathbb{H} * \otimes n} \rightarrow 0 \text { as } l \rightarrow \infty .
$$

By polarization of the identity, $\|\tilde{\alpha}\|_{L^{2}}^{2}=T^{n}\|\alpha\|_{n}^{2} / n$ !, it follows that

$$
(\tilde{\alpha}, \tilde{\beta})_{L^{2}}=\frac{T^{n}}{n !}(\alpha, \beta)_{\mathbb{H} * \otimes n} \text { for all } \alpha, \beta \in \mathbb{H}^{* \otimes n}
$$

Moreover, if $\alpha \in \mathbb{H}^{* \otimes n}$ and $\beta \in \mathbb{H}^{* \otimes m}$ with $m \neq n$, by the orthogonality of the finite dimensional approximations, $\tilde{\alpha}_{P_{l}}$ and $\tilde{\beta}_{P_{l}}$, we have that $(\tilde{\alpha}, \tilde{\beta})_{L^{2}}=0$.

Corollary 9.9 (Itô's isometry) Suppose that $\alpha=\left\{\alpha_{n}\right\}_{n=0}^{\infty} \in \oplus_{n=0}^{\infty} \frac{T^{n}}{n !} \mathbb{H}^{* \otimes n}$, i.e. $\alpha_{n} \in$ $\mathbb{H}^{* \otimes n}$ for all $n$ such that

$$
\|\alpha\|_{T}^{2}=\sum_{n=0}^{\infty} \frac{T^{n}}{n !}\left\|\alpha_{n}\right\|_{n}^{2}<\infty .
$$

Then $\tilde{\alpha}:=\sum_{n=0}^{\infty} \tilde{\alpha}_{n}$ is $L^{2}(\mathbf{P})$-convergent and the map,

$$
\bigoplus_{n=0}^{\infty} \frac{T^{n}}{n !} \mathbb{H}^{* \otimes n} \ni \alpha \mapsto \tilde{\alpha} \in L^{2}(\mathbf{P})
$$

is an isometry, where $\mathbf{P}$ is the probability measure used in describing the law of $\{b(t)\}_{t \geq 0}$.

9.2 The stochastic Taylor map

Let $b(t)=\left(B(t), B_{0}(t)\right) \in \mathfrak{g}$ and $g(t) \in G$ be the Brownian motions introduced at the start of Sect. 4. We are going to use the results of the previous subsection with $\mathbb{H}=$ $\mathfrak{g}_{C M}, \mathbb{W}=\mathfrak{g}$, and $b(t)=\left(B(t), B_{0}(t)\right)$. Let $f \in \mathcal{H}_{T}^{2}(G)$ and $\alpha_{f}:=\mathcal{T}_{T} S_{T} f \in J_{T}^{0}\left(\mathfrak{g}_{C M}\right)$. The following theorem is a (precise) restatement of Theorem 1.9.

Theorem 9.10 For any $f \in \mathcal{H}_{T}^{2}(G)$

$$
f(g(T))=\tilde{\alpha}_{f},
$$


where $\tilde{\alpha}_{f}$ was introduced in Corollary 9.9. (The right hand side of Eq. (1.8) is to be interpreted as $\tilde{\alpha}_{f}$.)

Proof First suppose that $f$ is a holomorphic polynomial and $P \in \operatorname{Proj}(W)$ so that $\pi_{P} \in \operatorname{Proj}(\mathfrak{g})$. Then by Itô's formula,

$$
f\left(g_{P}(T)\right)=f(\mathbf{e})+\int_{0}^{T}\left\langle D f\left(g_{P}(t)\right), d \pi_{P} b(t)\right\rangle .
$$

Iterating this equation as in the proof of [3, Proposition 5.2], if $N \in \mathbb{N}$ is sufficiently large, then

$$
\begin{aligned}
f\left(g_{P}(T)\right) & =f(\mathbf{e})+\sum_{n=1}^{N} \int_{0 \leq s_{1} \leq s_{2} \leq \cdots \leq s_{n} \leq T}\left\langle D^{n} f(\mathbf{e}), d \pi_{P} b\left(s_{1}\right) \otimes \cdots \otimes d \pi_{P} b\left(s_{n}\right)\right\rangle \\
& =f(\mathbf{e})+\sum_{n=1}^{N}\left[D^{n} f(\mathbf{e})\right]_{\pi_{P}}^{\sim} .
\end{aligned}
$$

We now replace $P$ by $P_{k} \in \operatorname{Proj}(W)$ with $P_{k} \uparrow I$ in this identity. Using Propositions 4.12 and 9.8, we may now pass to the limit as $k \rightarrow \infty$ in order to conclude,

$$
f(g(T))=f(\mathbf{e})+\sum_{n=1}^{N}\left[D^{n} f(\mathbf{e})\right]^{\tilde{\alpha}}=\tilde{\alpha}_{f} .
$$

Now suppose that $f \in \mathcal{H}_{T}^{2}(G)$. By Theorem 7.1 we can find a sequence of holomorphic polynomials $\left\{f_{n}\right\}_{n=1}^{\infty} \subset \mathcal{P}$ such that

$$
\mathbb{E}\left|f(g(T))-f_{n}(g(T))\right|^{2}=\left\|f-f_{n}\right\|_{L^{2}\left(v_{T}\right)}^{2} \rightarrow 0 \text { as } n \rightarrow \infty .
$$

The isometry property of the Taylor and skeleton maps (Theorem 6.10 and Corollary 8.3), shows that $\alpha_{f_{n}} \rightarrow \alpha_{f}$ in $J_{T}^{0}$ and therefore by Corollary $9.9 \tilde{\alpha}_{f_{n}} \rightarrow \tilde{\alpha}_{f}$ as $n \rightarrow \infty$. Hence we may pass to the limit in Eq. (9.7) applied to the sequence $f_{n}(g(T))=\tilde{\alpha}_{f_{n}}$, to complete the proof of Eq. (9.6).

\section{Future directions and questions}

In this last section, we wish to speculate on a number of ways that the results in this paper might be generalized.

(1) It should be possible to remove the restriction on $\mathbf{C}$ being finite dimensional, i.e. we expect much of what have done in this paper to go through when $\mathbf{C}$ is replaced by a separable Hilbert space. In doing so one would have to modify the finite dimensional approximations used in our construction to truncate $\mathbf{C}$ as well. 
(2) We also expect that the level of non-commutativity of $G$ may be increased. To be more precise, under suitable hypothesis it should be possible to handle more general graded nilpotent Lie groups.

(3) Open questions:

(a) as we noted in Remark 5.13 we do not know if $\mathcal{A}_{T}^{p}=\mathcal{H}_{T}^{p}(G)$. It might be easier to try to answer this question for $p=2$.

(b) give an intrinsic characterization of $\mathcal{H}_{T}^{2}(G)$ as in Shigekawa [25] in terms of functions in $L^{2}\left(v_{T}\right)$ solving a weak form of the Cauchy-Riemann equations.

Acknowledgments We are grateful to Professor Malliavin whose question during a workshop at the Hausdorff Institute (Bonn, Germany) led us to include a section on a holomorphic chaos expansion.

Open Access This article is distributed under the terms of the Creative Commons Attribution Noncommercial License which permits any noncommercial use, distribution, and reproduction in any medium, provided the original author(s) and source are credited.

\section{References}

1. Bargmann, V.: On a Hilbert space of analytic functions and an associated integral transform. Comm. Pure Appl. Math. 14, 187-214 (1961) (MR 28 \#486)

2. Cecil, M.: The Taylor map on complex path groups. J. Funct. Anal. 254, 318-367 (2008)

3. Driver, B.K.: On the Kakutani-Itô-Segal-Gross and Segal-Bargmann-Hall isomorphisms. J. Funct. Anal. 133(1), 69-128 (1995)

4. Driver, B.K., Gordina, M.: Heat kernel analysis on infinite-dimensional Heisenberg groups. J. Funct. Anal. 255, 2395-2461 (2008)

5. Driver, B.K., Gordina, M.: Integrated Harnack inequalities on Lie groups, p. 41. http://www.citebase. org/abstract?id=oai:arXiv.org:0711.4392 (2008, preprint)

6. Driver, B.K., Gross, L.: Hilbert spaces of holomorphic functions on complex Lie groups, New trends in stochastic analysis (Charingworth, 1994), pp. 76-106. World Sci. Publishing, River Edge (1997) [MR MR1654507 (2000h:46029)]

7. Driver, B.K., Gross, L., Saloff-Coste, L.: Surjectivity of the Taylor map for complex nilpotent lie groups tbd, 1-19 (2007, preprint)

8. Driver, B.K., Hall, B.C.: Yang-Mills theory and the Segal-Bargmann transform. Comm. Math. Phys. 201(2), 249-290 (1999) (MR 2000c:58064)

9. Fock, V.: Verallgemeinerung und Lösung der Diracschen statistischen Gleichung. Z. Phys. 49, 339_ 357 (1928)

10. Gordina, M.: Heat kernel analysis and Cameron-Martin subgroup for infinite dimensional groups. J. Funct. Anal. 171(1), 192-232 (2000)

11. Gordina, M.: Holomorphic functions and the heat kernel measure on an infinite-dimensional complex orthogonal group. Potential Anal. 12(4), 325-357 (2000)

12. Gordina, M.: Taylor map on groups associated with a $\mathrm{II}_{1}$-factor. Infin. Dimens. Anal. Quantum Probab. Relat. Top. 5(1), 93-111 (2002)

13. Gross, L., Malliavin, P.: Hall's transform and the Segal-Bargmann map. Itô's stochastic calculus and probability theory, pp. 73-116. Springer, Tokyo (1996) [MR MR1439519 (98j:22010)]

14. Hall, B.C., Sengupta, A.: The Segal-Bargmann transform for path-groups. J. Funct. Anal. 152(1), 220254 (1998)

15. Hervé, M.: Analyticity in infinite-dimensional spaces. de Gruyter Studies in Mathematics, vol. 10. Walter de Gruyter \& Co., Berlin (1989) [MR MR986066 (90f:46074)]

16. Hida, T., Kuo, H.-H., Potthoff, J., Streit, L.: White noise. Mathematics and its Applications, vol. 253. Kluwer, Dordrecht (1993). [An infinite-dimensional calculus. MR MR1244577 (95f:60046)]

17. Hille, E., Phillips, R.S.: Functional analysis and semi-groups, American Mathematical Society, Providence, R. I., 1974, Third printing of the revised edition of 1957, vol. XXXI. American Mathematical Society Colloquium Publications [MR MR0423094 (54 \#11077)] 
18. Kondratiev, Y.G.: Spaces of entire functions of an infinite number of variables connected with a rigging of Fock space, Spectral analysis of differential operators. Akad. Nauk Ukrain, pp. 18-37, 132. SSR Inst. Mat., Kiev (1980) [MR MR642527 (84e:46043)]

19. Kondratiev, Y.G., Leukert, P., Potthoff, J., Streit, L., Westerkamp, W.: Generalized functionals in Gaussian spaces: the characterization theorem revisited. J. Funct. Anal. 141(2), 301-318 (1996) [MR MR1418508 (97j:60070)]

20. Lempert, L.: Holomorphic functions on (generalized) loop spaces. Math. Proc. R. Ir. Acad. 104A(1), 35-46 (2004) (electronic) [MR MR2139508 (2006d:58008)]

21. Obata, N.: White noise calculus and Fock space. Lecture Notes in Mathematics, vol. 1577. Springer, Berlin (1994) [MR MR1301775 (96e:60061)]

22. Pickrell, D.: Measures on infinite-dimensional Grassmann manifolds. J. Funct. Anal. 70(2), 323-356 (1987) [MR MR874060 (88d:58017)]

23. Pickrell, D.: Invariant measures for unitary groups associated to Kac-Moody Lie algebras. Mem. Am. Math. Soc. 146(693), x+125 (2000) [MR MR1686655 (2000m:22023)]

24. Segal, I.E.: Mathematical problems of relativistic physics, With an appendix by George W. Mackey. Lectures in Applied Mathematics (proceedings of the Summer Seminar, Boulder, Colorado, vol. 1960. American Mathematical Society, Providence (1963) [MR MR0144227 (26 \#1774)]

25. Shigekawa, I.: Itô-Wiener expansions of holomorphic functions on the complex Wiener space, Stochastic analysis, pp. 459-473. Academic Press, Boston (1991)

26. Sugita, H.: Properties of holomorphic Wiener functions - skeleton, contraction, and local Taylor expansion, Probab. Theory Related Fields 100(1), 117-130 (1994) [MR MR1292193 (96h:60092)]

27. Sugita, H.: Regular version of holomorphic Wiener function. J. Math. Kyoto Univ. 34, 849-857 (1994)

28. Titchmarsh, E.C.: The Theory of Functions, 2nd edn. Oxford University Press, New York (1968) 